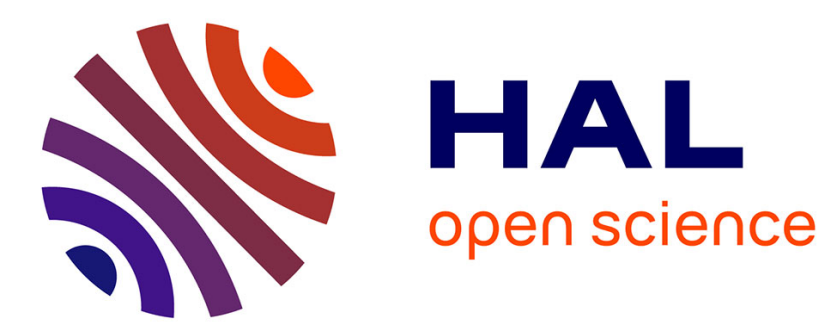

\title{
Mécanismes d'anisotropie dans la gravure du silicium en plasma SF6. Modèle de gravure
}

\author{
B. Petit, Jean Pelletier
}

\section{To cite this version:}

B. Petit, Jean Pelletier. Mécanismes d'anisotropie dans la gravure du silicium en plasma SF6. Modèle de gravure. Revue de Physique Appliquée, 1986, 21 (6), pp.377-399. 10.1051/rphysap:01986002106037700 . jpa-00245456

\section{HAL Id: jpa-00245456 https://hal.science/jpa-00245456}

Submitted on 1 Jan 1986

HAL is a multi-disciplinary open access archive for the deposit and dissemination of scientific research documents, whether they are published or not. The documents may come from teaching and research institutions in France or abroad, or from public or private research centers.
L'archive ouverte pluridisciplinaire HAL, est destinée au dépôt et à la diffusion de documents scientifiques de niveau recherche, publiés ou non, émanant des établissements d'enseignement et de recherche français ou étrangers, des laboratoires publics ou privés. 
Classification

Physics Abstracts

$81.60 \mathrm{C}-52.40 \mathrm{H}-82.65 \mathrm{~J}$

\title{
Mécanismes d'anisotropie dans la gravure du silicium en plasma $\mathbf{S F}_{6}$ • Modèle de gravure
}

\author{
B. Petit et J. Pelletier \\ Laboratoire de Physique des Milieux Ionisés, UA C.N.R.S. no 844, \\ CNS-CNET, B.P. 98, 38243 Meylan Cedex, France
}

(Reçu le 27 novembre 1985, révisé le 31 janvier 1986, accepté le 14 mars 1986)

\begin{abstract}
Résumé. - Les mécanismes de gravure du silicium en plasma de $\mathrm{SF}_{6}$ sont étudiés aux basses pressions et aux faibles énergies ioniques dans un Plasma Multipolaire Micro-onde. L'analyse des profils obtenus sur des motifs gravés et celle des produits de réaction détectés par spectrométrie de masse montre comment la vitesse de gravure et l'anisotropie évoluent en fonction de la pression. En particulier, on observe une transition régime isotroperégime anisotrope pour une valeur critique de la pression. Pour expliquer les résultats expérimentaux obtenus, une nouvelle interprétation des mécanismes de surface intervenant en gravure plasma est proposée. Afin de rendre cohérents les modèles existants, il est nécessaire de leur adjoindre trois hypothèses supplémentaires, à savoir : i) existence de fortes interactions répulsives entre atomes de fluor adsorbés proches voisins; ii) diffusion en surface des espèces adsorbées; iii) modèle d'adsorption multicouches pour le système $\mathrm{Si} / \mathrm{F}$. Le traitement analytique du modèle met en évidence que la vitesse de gravure est limitée par la pression partielle de fluor atomique dans le plasma, tandis que l'anisotropie est contrôlée par la densité de courant ionique.
\end{abstract}

\begin{abstract}
The mechanisms responsible for etching of silicon in low pressure $\mathrm{SF}_{6}$ plasma under low energy ion impact are studied using Microwave Multipolar Plasmas. Experimental results using both mass spectrometric and etch profile analysis show the evolution of anisotropy and etch rate as a function of pressure. In particular, a transition to perfect anisotropy is observed below a threshold pressure. To account for these experimental results, a new interpretation is proposed for the surface processes observed in plasma etching. To make the current models consistent, it is necessary to add three following assumptions : i) large lateral repulsive interactions between chemisorbed fluorine atoms in nearest neighbour positions; ii) chemisorbed fluorine atom diffusion on the silicon surface; iii) multilayer adsorption for the $\mathrm{Si} / \mathrm{F}$ system. The analytical treatment of the model points out that the etch rate is limited by the partial pressure of atomic fluorine, and that the anisotropy is controlled by the ion current density.
\end{abstract}

\section{Introduction.}

La tendance vers la miniaturisation toujours plus poussée des circuits intégrés confère à l'anisotropie de gravure une importance croissante. Cette anisotropie, condition principale du respect des cotes imposées par le concepteur, devient donc l'aspect primordial de l'étape de gravure et il est clair que sa maîtrise ne peut être atteinte que par une compréhension aussi totale que possible des mécanismes de gravure.

Jusqu'à présent, l'anisotropie est obtenue par un choix judicieux soit des gaz ou mélanges de gaz, soit de la technique de giavure elle-même (gravure par faisceaux d'ions réactifs par exemple). Dans les deux cas, le principe consiste à limiter ou supprimer la gravure latérale spontanée due aux espèces neutres et à privilégier la gravure induite par le bombardement ionique. Dans l'exemple de la gravure par faisceaux d'ions réactifs, seule subsiste la gravure induite par les ions réactifs d'énergie et d'orientation contrôlées. En plasma, plusieurs voies sont offertes pour maîtriser l'anisotropie : 1) choix de gaz peu ou pas réactifs conduisant à des cinétiques de réaction spontanée faibles voire nulles (par exemple l'hydrogène pour la gravure du silicium [1]);2) choix de gaz ou de mélanges réactifs mais tels que l'attaque de la paroi latérale soit bloquée par passivation. Cette passivation peut être due, entre autres, à la pulvérisation des bords de masque (RIE haute énergie), aux dépôts de carbone ou de polymères fluorocarbonés sur les surfaces non soumises au bombardement des ions [2], etc... En fait, ces procédés, aussi habiles et efficaces soient-ils, n'apparaissent que comme des palliatifs, souvent difficiles à établir et à maîtriser. 
En présence de plasma, les deux processus de gravure, spontané et induit, sont généralement présents. En l'absence de phénomène de passivation des parois, la surgravure sous le masque, ou gravure latérale, est le résultat de la seule gravure spontanée alors que la gravure verticale est la résultante de la gravure spontanée et de la gravure induite. Pour étudier et comprendre ces mécanismes, il est nécessaire de s'appuyer sur des données expérimentales sûres afin de pouvoir avancer des hypothèses sur les mécanismes réactionnels compatibles avec celles-ci.

L'objet de cette étude est donc de suggérer certaines hypothèses découlant directement, pour la plupart d'entre elles, d'observations expérimentales et de proposer un modèle pour la gravure plasma. Le plan de l'étude est le suivant : la section 2 présente un rappel succinct des différents modèles de gravure spontanée et induite existant à ce jour. Dans la section 3, on montre comment l'analyse des produits de réaction de la gravure du silicium en plasma $\mathrm{SF}_{6}$ et l'observation simultanée des profils de gravure mettent clairement en évidence une transition régime isotrope-régime anisotrope. Ces observations suggèrent un ensemble d'hypothèses sur les mécanismes réactionnels qui sont présentées dans la section 4. A partir de celles-ci, la construction d'un nouveau modèle de gravure et son développement analytique sont effectués dans la section 5. Les conséquences qui en découlent pour la gravure sont discutées section 6 et certaines confirmations expérimentales apportées dans la dernière section.

\section{Les modèles de gravure classiques.}

A ce jour, de nombreux modèles ont été proposés, tant pour la gravure spontanée que pour la gravure induite.

Le modèle le plus répandu est celui de Winters, Coburn et Chuang (W.C.C.) dont le canevas est le suivant [3] :

1) L'adsorption, étape préalable à la réaction de gravure, s'effectue par l'intermédiaire d'états précurseurs [4] qui influencent fortement la cinétique de gravure.

2) Des phénomènes identiques à ceux que l'on connaît bien dans les réactions d'oxydation interviennent, qui sont de nature à influencer voire régir les réactions de gravure, à savoir : a) la gravure s'effectue vraisemblablement à partir d'une surface reconstruite; b) les mécanismes mis en jeu sont de type de ceux décrits par Mott-Cabrera pour l'oxydation et impliquent l'échange d'anions et de cations par substitution et diffusion sous l'effet de champs électriques.

3) Aucune gravure spontanée n'est observée si la chimisorption n'excède pas la monocouche.

4) La gravure spontanée conduit à des produits de réaction saturés tels que $\mathrm{SiF}_{4}$ par exemple, et non $\operatorname{SiF}_{x}(x<4)$.

5) L'énergie d'activation nécessaire à la réaction de gravure dépend du flux et de la nature des espèces incidentes.

Le modèle de gravure spontanée, proposé par Flamm et Donnelly [5] est beaucoup plus descriptif que le précédent et plus spécifique aussi, puisqu'il concerne uniquement la gravure du silicium par les atomes de fluor. Il fait intervenir une fluoruration du silicium par étapes ainsi que schématisée sur la figure 1 . La cinétique est alors contrôlée par l'étape I (ou II), qui apparaît comme le processus limitant. Une fois rompues les liaisons stables de la surface fluorée, les étapes de fluoruration successives sont rapides.

A partir du schéma de la figure 1, il est aisé de formuler le canevas conduisant à ce modèle :

1) Adsorption d'une couche chimisorbée stable $\mathrm{SiF}_{2}$. Cette hypothèse implique nécessairement, suivant l'orientation cristalline, une reconstruction de la surface.

2) Poursuite de la fluoruration du silicium conduisant à deux processus concurrents : a) désorption de $\mathrm{SiF}_{2}$ par rupture des liaisons $\mathrm{Si}-\mathrm{Si}$ (substitution par le fluor); b) formation puis désorption de $\mathrm{SiF}_{4}$.

3) La gravure spontanée telle que décrite précédemment n'est possible que pour des recouvrements en fluor dépassant la monocouche.

Toutefois, ces deux modèles sont muets quant au rôle que peuvent jouer les ions en gravure plasma. Or, d'une manière générale, les surfaces qui sont soumises à des flux simultanés d'espèces neutres réactives et

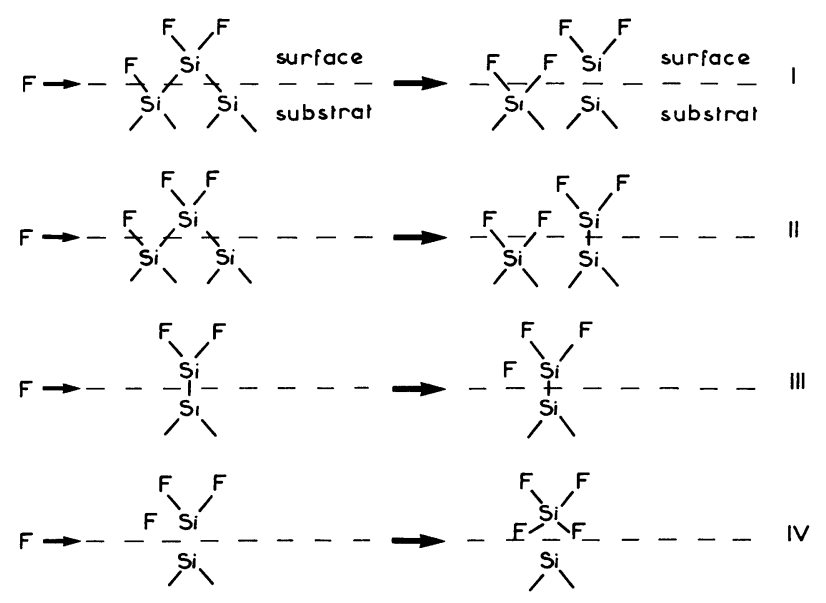

Fig. 1. - Mécanisme de gravure du silicium par les atomes de fluor (d'après Donnelly et Flamm [5]). I. - Rupture simultanée des liaisons $\mathrm{Si}-\mathrm{Si}$ conduisant à $\mathrm{SiF}_{2}$ gazeux, II. - Fluoruration du silicium conduisant à $\mathrm{SiF}_{2}$ chimisorbé, III et IV. - Fluorurations successives conduisant à $\mathrm{SiF}_{4}$ gazeux.

[Mechanism for the etching of silicon by fluorine atoms (from Donnelly and Flamm [5]). I. - Concerted reaction break bands, resulting in gaseous $\mathrm{SiF}_{2}$, II. - Silicon fluorination results in chemisorbed difluorosilicon $\mathrm{SiF}_{2}$, III and IV. - Further fluorination of bound radicals liberates gaseous $\mathrm{SiF}_{4}$.] 
d'ions énergétiques sont érodées plus rapidement que celles soumises aux seules espèces neutres. Cette observation peut être vérifiée quotidiennement en plasma car il n'est pas d'exemple où la vitesse de gravure latérale soit supérieure à la vitesse de gravure verticale. Parallèlement, l'effet du bombardement ionique sur l'érosion chimique a pu être mesuré quantitativement dans de nombreuses expériences effectuées hors milieu plasma et utilisant des faisceaux d'ions [6-9].

Si l'accroissement de la vitesse de gravure induite par le bombardement ionique est universellement reconnu, aucun consensus général ne se dégage quant à la manière dont le bombardement ionique accélère la cinétique réactionnelle sur la surface. $\mathrm{Si}$, comme pour la gravure spontanée, le processus peut se décomposer en trois étapes - adsorption, réaction, désorption le mécanisme d'interaction entre la surface et les ions reste une question ouverte.

A ce jour, trois modèles de gravure induite par les ions ont été proposés :

1) L'érosion ionique chimiquement activée (chemically enhanced physical sputtering). - Ce modèle est dû à Mauer et al. [10] qui ont constaté expérimentalement que la vitesse de gravure observée est supérieure à la vitesse d'érosion ionique purement mécanique et à la vitesse d'érosion chimique spontanée. Ils en ont déduit que les espèces $\operatorname{SiF}_{x}$ adsorbées sur la surface présentent un taux d'érosion ionique nettement supérieur à celui du silicium, et ce en raison d'une énergie de liaison moindre de ces espèces. Dans cette hypothèse, l'augmentation de la vitesse de gravure est donc tout simplement due à une augmentation du taux d'érosion ionique.

2) L'érosion chimique activée par les défauts induits (damage induced chemical reaction). - Cette interprétation suggérée par Coburn et Winters [6] a été ensuite reprise et développée par Flamm et Donnelly [5]. Elle suppose que les défauts induits dans le réseau à la surface du silicium par le bombardement ionique favorise l'adsorption dissociative des espèces réactives, augmentant ainsi la cinétique de gravure.

3) L'érosion chimique induite (chemical sputtering). Dans ce dernier modèle, proposé par Tu, Chuang et Winters [7], les auteurs suggèrent que le bombardement induit sur la surface une réaction entre deux espèces $\mathrm{SiF}_{2}$ chimisorbées pour donner Si et $\mathrm{SiF}_{4}$ qui, faiblement lié à la surface, désorbe spontanément.

Finalement, ces modèles proposés aussi bien pour la gravure spontanée que pour la gravure induite ne sont incompatibles ni entre eux, ni avec les résultats expérimentaux connus à ce jour. Ils sont même relativement complémentaires. Pour la gravure spontanée, le modèle W.C.C. insiste sur les mécanismes alors que le modèle de Donnelly et Flamm est plutôt descriptif. Pour la gravure induite, les différents modèles proposés, dont deux par les mêmes auteurs s'appliquent en fait à des étapes fondamentales différentes du processus d'érosion chimique.
La grande diversité des modèles proposés illustre la difficulté d'une part à trancher entre les différentes hypothèses déduites des résultats expérimentaux connus, d'autre part à relier de façon cohérente gravure spontanée et gravure induite. Cela nécessite en fait de pouvoir répondre à un certain nombre de questions restées en suspens : i) quel est le mécanisme prépondérant responsable de la gravure induite ? Certains mécanismes faisant appel à un bombardement d'ions énergétiques $(>100 \mathrm{eV})$, la gravure induite subsiste-t-elle à basse énergie ? ii) quel est le lien entre gravure spontanée et gravure induite ? iii) quelles sont les hypothèses sur les mécanismes réactionnels les plus consistantes avec les résultats expérimentaux ? La réponse à ces questions ne peut provenir que de résultats expérimentaux clairs permettant de choisir, parmi les différentes hypothèses évoquées, les mieux adaptées. Telle est la démarche que nous avons adoptée et que nous allons mettre en œuvre.

\section{Gravure du silicium en plasma $\mathrm{SF}_{6}$ : résultats expé- rimentaux.}

Les résultats expérimentaux recherchés doivent répondre aussi clairement que possible aux incertitudes soulevées par les modèles rappelés dans la section précédente. Pour cela, une approche intéressante consiste à suivre l'évolution des cinétiques de gravure spontanée et induite en fonction d'un seul paramètre. Leurs cinétiques respectives sont en réalité parfaitement représentées par celle de la vitesse d'érosion verticale $V_{\mathrm{v}}$ et celle de l'anisotropie $A$ définie par la relation (1) :

$$
A=1-\frac{V_{1}}{V_{v}}
$$

où $V_{1}$ est la vitesse de gravure latérale spontanée.

Les Plasmas Multipolaires Micro-onde (PMM), qui permettent de dissocier la production du plasma de son interaction avec le substrat à graver et dont les caractéristiques peuvent être facilement déterminées sont tout à fait adaptés à cette étude $[11,12]$. Le dispositif expérimental utilisé est schématisé sur la figure 2. Le réacteur de gravure associe une excitation microonde localisée et un confinement magnétique multipolaire du plasma. Si le libre parcours moyen des ions est de l'ordre de grandeur des dimensions de l'enceinte, ou plus grand, la diffusion s'effectue dans l'ensemble de la structure multipolaire pour finalement donner un plasma homogène de caractéristiques similaires à celles des plasmas multipolaires classiques.

Dans notre dispositif expérimental, la source microonde localisée (Fig. 3) utilise la résonance cyclotronique électronique [13] (RCE). La puissance microonde $(2,45 \mathrm{GHz})$ délivrée par un générateur continu $(1,5 \mathrm{~kW})$ est transportée par un guide d'ondes équipé d'un adaptateur d'impédance puis injectée dans le plasma à travers une fenêtre d'alumine. Le champ magnétique de 875 gauss nécessaire pour obtenir la RCE est produit par une bobine placée à ce niveau. Le 


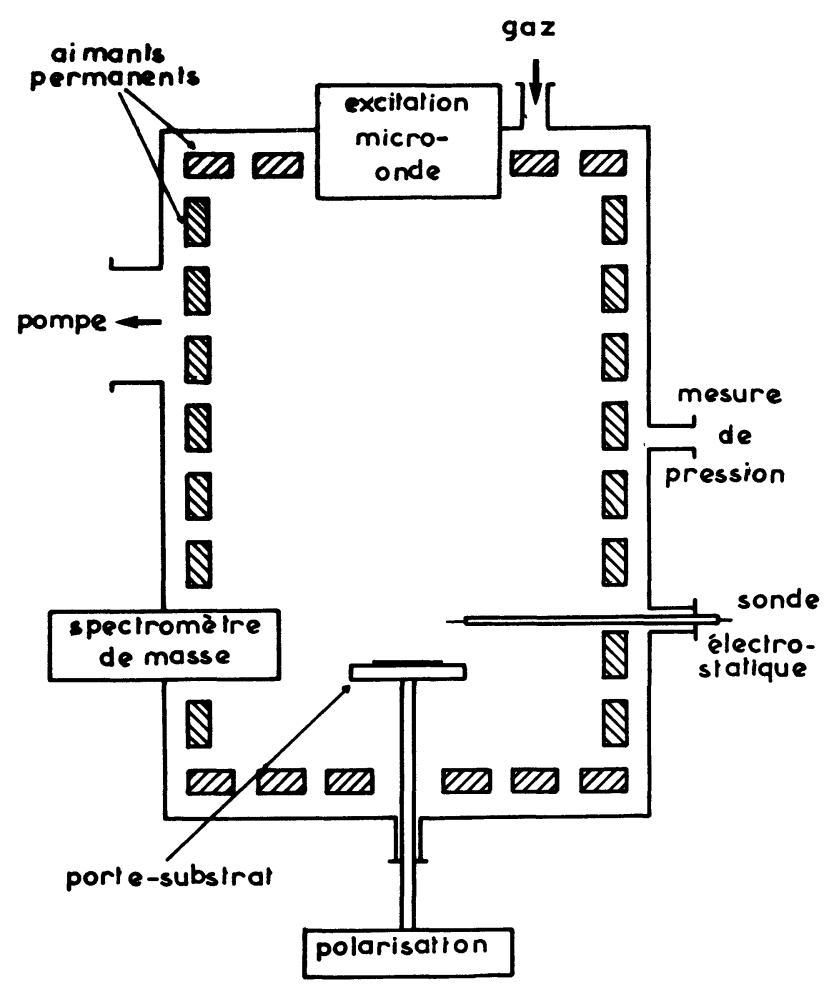

Fig. 2. - Représentation schématique du dispositif expérimental associant une excitation micro-onde à une structure de confinement magnétique multipolaire.

[Schematic representation of the experimental set-up combining a localized microwave discharge and multipolar magnetic confinement.]

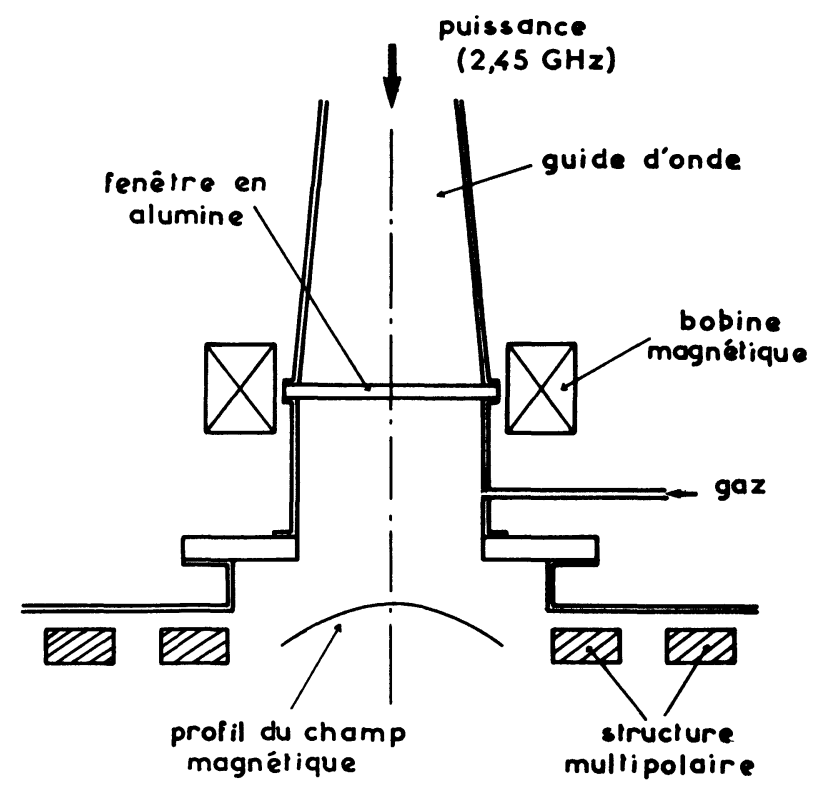

Fig. 3. - Schéma de la source micro-onde localisée RCE.

[Schematic representation of the ECR microwave plasma source.] plasma créé dans la source diffuse dans une structure multipolaire cylindrique longue de $70 \mathrm{~cm}$ et de $40 \mathrm{~cm}$ de diamètre. Le porte-échantillons de $10 \mathrm{~cm}$ de diamètre et la sonde de Langmuir cylindrique utilisée pour la caractérisation du plasma sont situés à une distance de la bobine magnétique $(70 \mathrm{~cm})$ telle que le champ magnétique est négligeable dans cette zone de l'enceinte. Enfin, le réacteur est équipé d'un spectromètre de masse qui peut analyser les produits de réaction collectés à travers un dispositif d'extraction constitué d'une grille de silicium [14]. L'intérêt d'un tel dispositif réside dans ce que, si l'on applique à la grille une polarisation identique à celle de l'échantillon, elle subit le même processus de gravure que celui-ci. Les produits de réaction sont ainsi recueillis au plus près et immédiatement analysés sans avoir à subir de collisions avec les parois du réacteur ou les espèces présentes dans le plasma. Leur analyse en transmission peut être alors directement corrélée avec l'observation des profils de gravure.

La nature du gaz introduit et sa pression constituent quelques-uns des paramètres plasma qui influent directement sur la gravure. Dans le cas de la gravure $\mathrm{du}$ silicium, qui nous intéresse plus particulièrement, nous avons choisi l'hexafluorure de soufre $\mathrm{SF}_{6}$ car d'une part il conduit généralement à des cinétiques de gravure spontanée importantes, et d'autre part il ne provoque pas de passivation des parois pouvant masquer la gravure latérale [15]. Un autre paramètre important est l'énergie des ions bombardant le silicium. Nous avons choisi, pour cette étude, de la maintenir constante, et ce, à une valeur suffisamment faible afin d'une part d'éliminer les phénomènes d'érosion purement mécanique, d'autre part de réduire la création de défauts induits à la surface du silicium [16]. A puissance de décharge constante, la densité de plasma, sa température électronique et la densité de courant d'ions recueillie sur l'échantillon sont aisément mesurables. Enfin, la pression, qui gouverne la concentration de fluor atomique dans le plasma a été retenue comme paramètre.

L'étude expérimentale a été menée dans une gamme de pression de $\mathrm{SF}_{6}$ comprise entre $2 \times 10^{-5}$ torr et quelques $10^{-4}$ torr et une puissance micro-onde de 600 watts. Les variations de la température électronique et de la densité de plasma sont présentées sur la figure 4, ainsi que la densité de courant $j$ recueillie sur les substrats de silicium polarisés en continu à $-70 \mathrm{~V}$. L'évolution de la vitesse de gravure verticale $V_{\mathrm{v}} \mathrm{du}$ silicium et de l'anisotropie $A$ en fonction de la pression $\mathrm{de} \mathrm{SF}_{6}$ est indiquée sur la figure 5 .

Lorsque la pression de $\mathrm{SF}_{6}$ croît, la vitesse de gravure augmente de façon uniforme alors que l'anisotropie, telle que définie par la relation (1) présente un comportement très différent. L'augmentation de la vitesse de gravure est liée à celle de la densité de fluor atomique dans la décharge qui, elle, croît avec la pression de $\mathrm{SF}_{6}[17,18]$. En ce qui concerne l'anisotropie, alors que l'on pouvait s'attendre, pour des pressions 


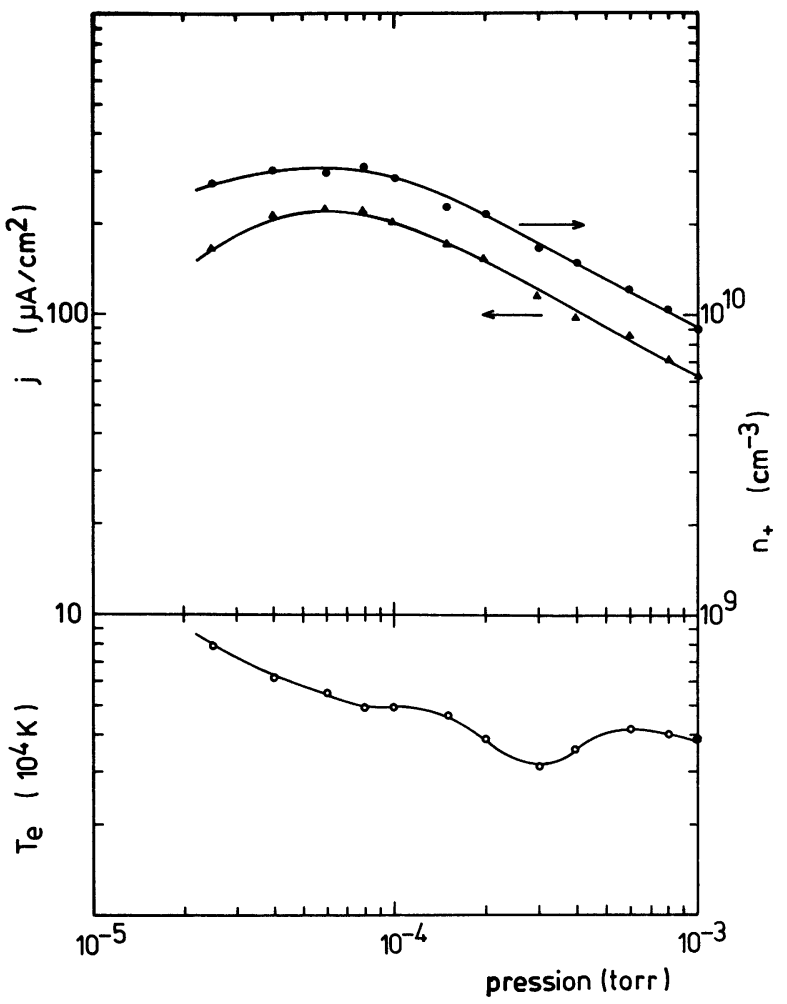

Fig. 4. - Evolution de la densité ionique, de la température électronique et de la densité de courant dans un plasma de $\mathrm{SF}_{6}$ en fonction de la pression de neutres pour une puissance UHF injectée de $600 \mathrm{~W}$.

[Ion density, electron temperature and ion current in an $\mathrm{SF}_{6}$ plasma as a function of pressure for an injected microwave power of $600 \mathrm{~W}$.]

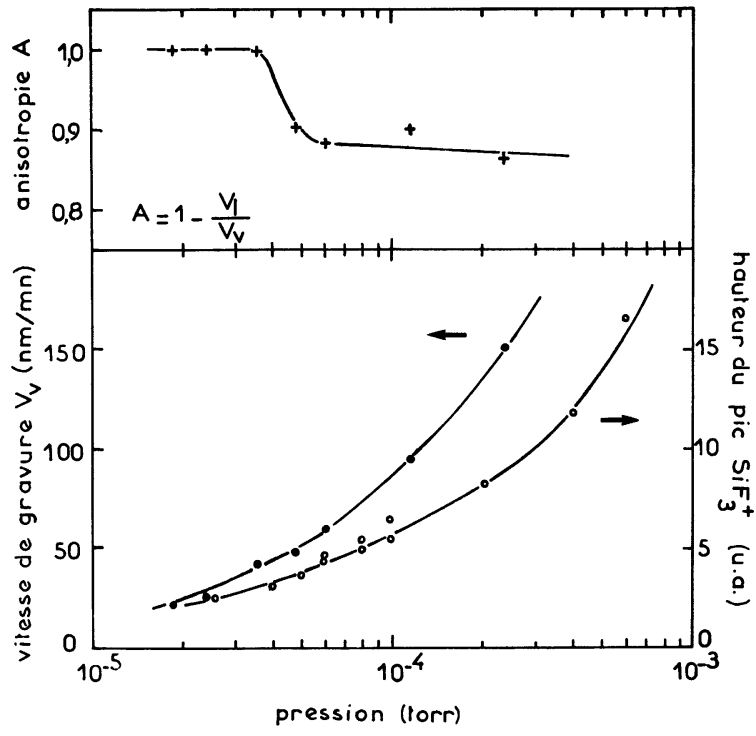

Fig. 5. - Evolution de la vitesse de gravure verticale $V_{\mathrm{v}} \mathrm{du}$ silicium, de l'anisotropie $A$ et de la hauteur du pic $\mathrm{SiF}_{3}^{+}$en fonction de la pression de $\mathrm{SF}_{6} . V_{1}$ est la vitesse de gravure latérale.

[Vertical etch rate $V_{v}$, anisotropy $A$ and $\mathrm{SiF}_{3}^{+}$peak intensity as a function of $\mathrm{SF}_{6}$ pressure. $V_{1}$ is the lateral etch rate.] de $\mathrm{SF}_{6}$ décroissantes, à une augmentation progressive due à la diminution du flux aléatoire d'espèces réactives par rapport au flux d'ions, on observe une variation brutale de l'anisotropie qui devient égale à l'unité $\left(V_{1}=0\right)$. La densité de courant d'ions reçue par l'échantillon étant quasiment constante dans le domaine de variation brutale de l'anisotropie, on en déduit donc que la gravure spontanée disparaît audessous d'une pression critique de fluor atomique $p_{\mathrm{c}}$ directement liée à la pression de $\mathrm{SF}_{6}$ [17].

L'analyse simultanée par spectrométrie de masse des produits de réaction issus de la grille de silicium montre que, toujours pour une polarisation de $-70 \mathrm{~V}$, le seul produit de réaction est $\mathrm{SiF}_{4}$ (molécule mère de l'ion $\mathrm{SiF}_{3}^{+}$) sauf aux pressions les plus basses où les pics 47 et 66 (en unités de masse atomique) montrent une légère croissance attribuée à la désorption de la molécule mère $\mathrm{SiF}_{2}$. La distinction entre $\mathrm{COF}_{2}$ et $\mathrm{SiF}_{2}$ a été effectuée en spectrométrie de masse en utilisant une méthode de coïncidence [19] (" semi-log matching method »). L'évolution du seul pic $\mathrm{SiF}_{3}^{+}$, telle qu'indiquée sur la figure 5 ne montre pas de corrélation entre l'anisotropie et la quantité de produit de réaction $\mathrm{SiF}_{4}$ désorbé. Par contre, l'évolution relative des pics 47,66 et 85 par rapport à la totalité des produits désorbés met en évidence (Fig. 6) l'apparition de produits de réaction non saturés en fluor lors du passage à l'anisotropie unité. Les enseignements que l'on peut tirer de ce résultat sont les suivants : i) la gravure spontanée, responsable du défaut d'anisotropie, apparaît seulement au-delà d'un recouvrement critique $\theta_{c}$ de fluor sur le silicium, recouvrement lié à la pression

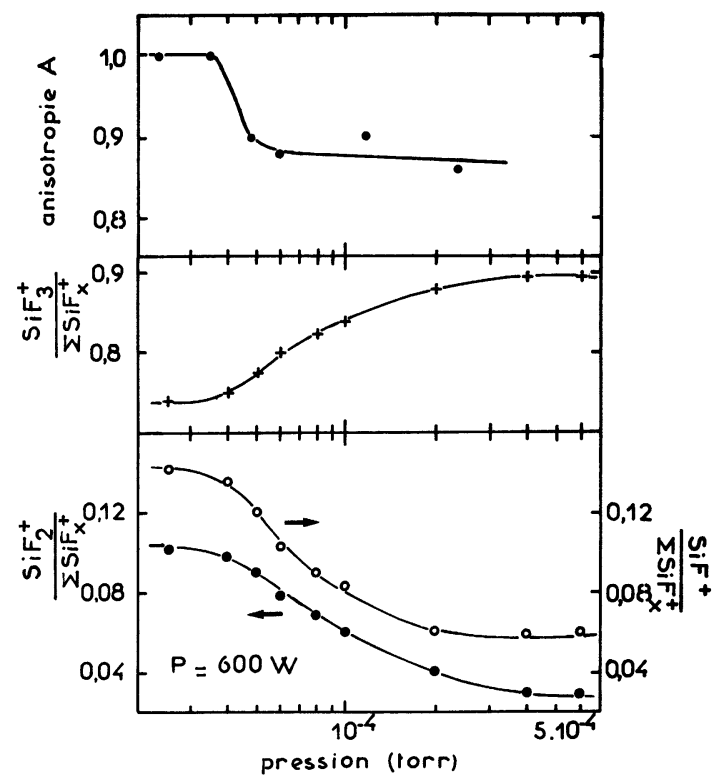

Fig. 6. - Evolution relative des pics $47\left(\mathrm{SiF}^{+}\right), 66\left(\mathrm{SiF}_{2}^{+}\right)$et $85\left(\mathrm{SiF}_{3}^{+}\right)$par rapport à la totalité des produits désorbés et de l'anisotropie observée en fonction de la pression de $\mathrm{SF}_{6}$.

[Mass spectrometric analysis of the reaction products : relative intensity of peaks $47\left(\mathrm{SiF}^{+}\right), 66\left(\mathrm{SiF}_{2}^{+}\right)$and $85\left(\mathrm{SiF}_{3}^{+}\right)$ as a function of $\mathrm{SF}_{6}$ pressure.] 
critique $p_{\mathrm{c}}$; ii) la disparition de la gravure spontanée s'accompagne d'une diminution relative $\mathrm{de}^{\mathrm{SiF}_{4}}$ dans les produits désorbés. Cette constatation semble indiquer que la gravure spontanée conduit au seul produit $\mathrm{SiF}_{4}$; iii) l'apparition de produits de réaction non saturés lors de la gravure induite témoigne d'une diminution du recouvrement du silicium en fluor.

L'ensemble des résultats expérimentaux présentés dans cette section a permis non seulement de mettre en évidence et d'analyser le passage gravure isotropegravure anisotrope, mais aussi de dissocier la contribution de la gravure induite anisotrope de celle de la gravure spontanée isotrope. L'exploitation de l'ensemble de ces résultats nous amène tout naturellement à proposer nos hypothèses sur les mécanismes réactionnels mis en jeu dans la gravure plasma.

\section{Hypothèses sur les mécanismes réactionnels}

Le modèle de gravure présenté dans cette section et développé dans les suivantes est un modèle spécifique de la gravure du silicium en plasma fluoré. Nous évoquerons succinctement à la fin de l'article les possibilités d'extension d'un tel modèle à d'autres systèmes chimiques. Les hypothèses que nous avançons s'appuient sur nos résultats expérimentaux mais aussi sur certains des résultats très nombreux de la littérature concernant le couple silicium-fluor. Certaines hypothèses des modèles présentés dans la section 2 sont reprises, puis précisées après analyse et discussion.

Dans notre schéma, nous distinguons deux étapes : l'étape de chimisorption du fluor sur le silicium, puis l'étape, spontanée ou induite, de formation des produits de réaction et de leur désorption.

La chimisorption du fluor sur la surface du silicium a pu être mise en évidence dans de nombreuses expériences, soit à partir de plasmas de gaz fluorés, soit même directement à partir de $\mathrm{F}_{2}, \mathrm{XeF}_{2}$ voire $\mathrm{SiF}_{4}$. Dans le cas des plasmas de gaz fluorés, il y a chimisorption directe du fluor atomique présent dans le plasma et provenant de la dissociation en volume du gaz utilisé. Dans l'autre cas, la fluoruration de la surface de silicium [3, 20,21] s'effectue par chimisorption dissociative du gaz pour aboutir sur la surface à un recouvrement d'espèces chimisorbées supposées être $\mathrm{SiF}_{2}$ $[7,20,22]$ et pouvant rester adsorbées pendant de longues périodes sur la surface du silicium [20, 22]. $\mathrm{Si}$ l'exposition au fluor est prolongée, la gravure spontanée du silicium apparaît avec formation de $\mathrm{SiF}_{4}$. On observe alors une incorporation du fluor en volume [3, 23, 24] sous forme chimisorbée [25] sur une profondeur qui augmente avec le temps d'exposition [23]. Si l'on s'intéresse maintenant aux premières étapes de la fluoruration, les expériences effectuées par UPS semblent indiquer que le mécanisme de fluoruration est séquentiel [26].

Compte tenu de ces résultats et de ceux que nous avons présentés dans la section précédente, il est alors possible de proposer un certain nombre d'hypothèses sur les mécanismes de gravure du silicium qui sont les suivantes :

1) L'adsorption s'effectue par un mécanisme séquentiel passant par les étapes schématisées sur la figure 7;

2) $\mathrm{SiF}_{4}$ est le seul produit de réaction de la gravure spontanée;

3) Le mécanisme réactionnel de la gravure spontanée est : $\mathrm{SiF}_{2}(\mathrm{ads})+\mathrm{SiF}_{2}(\mathrm{ads}) \rightarrow \mathrm{Si}+\mathrm{SiF}_{4}(\mathrm{gaz})(2)$;

4) La réaction (2) n'est possible qu'entre deux groupes $\mathrm{SiF}_{2}$ plus proches voisins;

5) Le bombardement ionique favorise sur la surface la formation des produits de réaction selon la réaction (2);

6) Le bombardement ionique induit la désorption des produits de réaction $\mathrm{SiF}_{x}$ autres que $\mathrm{SiF}_{4}(x<4)$.

Ces hypothèses apparaissent pour la plupart comme une déduction directe des observations expérimentales. En particulier, les hypothèses 5 et 6 font intervenir des mécanismes basse énergie autres que la pulvérisation par érosion ionique. Ce point a aussi été mis en évidence avec des ions de forte énergie en mesurant la dépendance angulaire de la vitesse de gravure du silicium soumis au bombardement d'un faisceau d'ions réactifs sous incidence variable $[9,27]$, dépendance angulaire qui diffère notablement de celle qu'on observe en érosion ionique de type purement mécanique $[3,9]$.

En fait, les seuls apports originaux par rapport aux modèles existants concernent le mécanisme séquentiel d'adsorption (Hypothèse 1) et la condition de réaction spontanée (Hypothèse 4). Ces deux hypothèses sont en réalité la clef du modèle car elles permettent d'apporter une explication plausible au phénomène caractéristique que constitue le passage à une anisotropie unité quand on descend au-dessous d'un seuil critique de
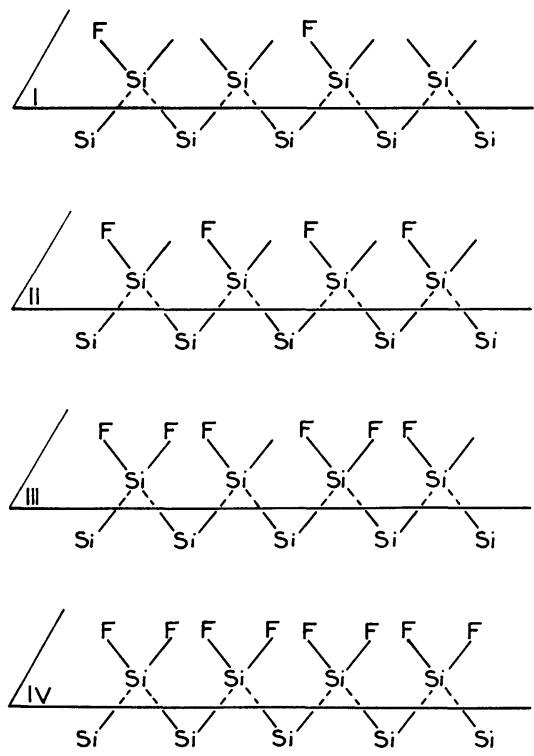

Fig. 7. - Mécanisme séquentiel de fluoruration du silicium.

[Sequential mechanism of the surface fluorination of silicon.] 
recouvrement en fluor du silicium. Toutefois, l'hypothèse 1 concernant le mécanisme séquentiel d'adsorption implique à son tour de nouvelles hypothèses, la première sur la nature des interactions entre atomes de fluor adsorbés, la seconde sur la diffusion en surface des espèces adsorbées.

Si l'on suppose que les interactions entre deux atomes adsorbés sont limitées aux seuls plus proches voisins, seule l'hypothèse de l'interaction répulsive permet d'expliquer le mécanisme d'adsorption adopté dans l'hypothèse 1 : si l'énergie d'interaction $\varepsilon$ est attractive (modèle d'Ising ferromagnétique), il s'établit, au-dessous d'une certaine température, un ordre à grande distance conduisant à une séparation de phase [25]. Au-delà de cette température, l'énergie thermique devient alors prépondérante devant l'énergie d'interaction, provoquant la transition vers une phase unique désordonnée. Dans le cas d'une énergie d'interaction $\varepsilon$ répulsive (modèle d'Ising antiferromagnétique), seule une phase continue apparaît sur la presque totalité du domaine de température et de recouvrement. Une région de phase ordonnée peut apparaître à basse température dans un domaine très limité de recouvrement [28]. Dans le cas de la figure 7, il est clair qu'un ordre à grande distance ne peut s'établir que pour les valeurs de recouvrement égales ou proches d'un multiple de 0,25 . Une autre possibilité pour aboutir à un mécanisme d'adsorption séquentiel similaire à celui représenté sur la figure 7 serait de considérer une énergie d'interaction répulsive entre proches voisins et une énergie d'interaction attractive entre seconds voisins, l'énergie d'interaction restant globalement attractive. Un tel modèle permet d'expliquer la formation de structures $p(2 \times 2)$ ou $c(2 \times 2)$, mais dans ce cas on observe à nouveau une séparation de phase [28]. Toutefois, un tel modèle qui implique, à côté d'une phase pauvre en fluor, l'existence d'une phase saturée en fluor, n'est guère compatible avec notre hypothèse 4 et la présence pendant de longues périodes sur la surface du silicium d'espèces adsorbées du type $\mathrm{SiF}_{2}[20,22]$. De plus, supprimer l'hypothèse 4 conduirait de fait à ne plus pouvoir avoir de gravure induite sans gravure spontanée, ni, par conséquent, d'obtenir une anisotropie unité.

La seconde hypothèse implicite de notre modèle concerne la diffusion en surface des espèces adsorbées. Cette diffusion est indispensable pour la cohérence de notre modèle et ce pour deux raisons. La première est que la diffusion latérale sur la surface est la condition sine qua non pour que le mécanisme séquentiel d'adsorption (Hypothèse 1) puisse s'effectuer et pour que l'ordre à grande distance et/ou à courte distance puisse s'établir sur la surface entre espèces adsorbées. Supposons en effet qu'il existe une température seuil, au-dessous de laquelle les adatomes ne pourraient pas diffuser latéralement sur la surface : dans ce cas, l'adsorption conduirait à un recouvrement aléatoire et la couche adsorbée serait figée [29, 30]. La seconde raison pour laquelle l'hypothèse d'une diffusion en surface est nécessaire est une conséquence directe de l'existence d'une anisotropie unité. En effet, en gravure plasma $\mathrm{SF}_{6}$ du silicium, l'absence de gravure spontanée ne peut être obtenue qu'en maintenant le recouvrement au-dessous d'une valeur critique. Si l'on suppose que toute la surface de silicium est soumise au flux aléatoire de fluor atomique, un tel recouvrement ne peut donc être maintenu qu'en supposant un flux par diffusion en surface vers les zones de plus faible recouvrement, c'est-à-dire les zones de gravure induite qui subissent le bombardement ionique.

Les résultats expérimentaux présentés dans la section 3 et le modèle proposé dans la présente section impliquent, comme on l'a vu, d'une part l'existence d'interactions répulsives entre adatomes de fluor, d'autre part la diffusion des espèces chimisorbées sur la surface. Dans la section 5 nous allons voir comment traduire analytiquement ces deux hypothèses et comment on aboutit logiquement au modèle de gravure proposé.

\section{Phase adsorbée : développement analytique du modèle.}

L'adsorption séquentielle du fluor sur le silicium tel qu'elle est schématisée sur la figure 7 peut se décomposer en deux étapes : la première correspond aux séquences I et II et aboutit à la formation d'une couche uniforme $\mathrm{SiF}$; la seconde correspond aux séquences III et $\mathrm{IV}$ et aboutit à la formation d'une couche $\mathrm{SiF}_{2}$. A ce stade du recouvrement, toutes les liaisons pendantes à la surface du silicium sont occupées par des atomes de fluor. Le recouvrement en fluor $\theta_{\mathbf{F}}$ est alors égal à l'unité. Si l'on poursuit la fluoruration $\left(\theta_{\mathrm{F}}>1\right)$, on incorpore alors (cf. Sect. 4) du fluor en volume avec formation de $\mathrm{SiF}_{3}$ suivant un mécanisme analogue suggéré par Donnelly et Flamm [5] (Fig. 1). Dans la suite de cette section, nous nous limiterons au cas où le recouvrement en fluor reste inférieur à l'unité.

Si l'on admet que le mécanisme d'adsorption séquentiel est rendu possible par la diffusion en surface des adatomes de fluor, l'hypothèse d'interactions répulsives entre atomes de fluor adsorbés est équivalente en fait à l'hypothèse d'interactions répulsives entre " espèces " $\operatorname{SiF}_{x}(x=1$ puis $x=2)$ à la surface du silicium. Il en va de même pour leur diffusion qui est en fait assurée par celle des atomes de fluor. La formation de la couche $\mathrm{SiF}$ puis celle de la couche $\mathrm{SiF}_{2}$ 's'effectuent alors suivant un même modèle. Comme indiqué sur la figure 8 , à un recouvrement en fluor $0<\theta<0,5$ correspond un recouvrement en espèces $\mathrm{SiF} 0<\theta_{\mathrm{SiF}}<1$; à un recouvrement en fluor $0,5<\theta<1$ correspond un recouvrement en espèces $\mathrm{SiF}_{2} 0<\theta_{\mathrm{SiF}_{2}}<1$. Compte tenu des hypothèses 3 et 4 de notre modèle relatives aux conditions d'apparition de la gravure spontanée, nous nous intéresserons plus spécialement à la formation de la couche formée par les espèces $\mathrm{SiF}_{2}$. Toujours.d'après 

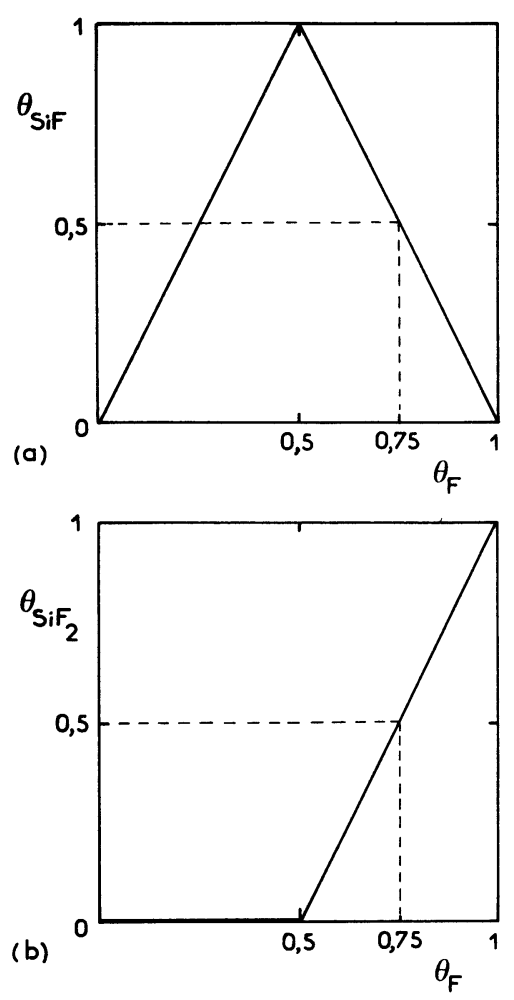

Fig. 8. - Evolution du taux de recouvrement en $\mathrm{SiF}$ et $\mathrm{SiF}_{2}$ en fonction du recouvrement en fluor.

[ $\mathrm{SiF}$ and $\mathrm{SiF}_{2}$ coverage evolution as a function of fluorine coverage on silicon.]

le modèle proposé, la formation de $\mathrm{SiF}_{4}$ volatil résulte de la réaction entre deux groupes $\mathrm{SiF}_{2}$ proches voisins. Le calcul du nombre de groupes $\mathrm{SiF}_{2}$ proches voisins qui apparaissent lors de la formation de la couche de $\mathrm{SiF}_{2}$ doit donc permettre de déterminer les conditions d'apparition de la gravure spontanée.

Pour ce calcul, nous utiliserons les notations suivantes : soit $A$ un site occupé par un groupe $\mathrm{SiF}_{2}$ et $\mathrm{O}$ un site inoccupé, soit AA une paire de sites proches voisins occupés, OA une paire de sites proches voisins dont seul l'un d'entre eux est occupé et $O O$ une paire de sites proches voisins inoccupés. Soient $N_{\mathrm{A}}, N_{\mathrm{O}}$ $N_{\mathrm{AA}}, N_{\mathrm{OA}}$ et $N_{\mathrm{Oo}}$ les nombres de sites ou paires de sites correspondants. Prenons pour origine des énergies le niveau d'énergie d'un site occupé $A$ entouré de sites inoccupés et soit $\varepsilon$ l'énergie d'interaction entre les deux sites occupés d'une paire AA. Le calcul du nombre de sites proches voisins occupés $N_{\mathrm{AA}}$, qui nous intéresse ici plus particulièrement, consiste d'abord à écrire l'équilibre entre sites voisins, puis de le relier au nombre de sites occupés $N_{\mathrm{A}}$. L'occupation de deux sites voisins est régie par l'équilibre suivant :

$$
\mathrm{AA}+\mathrm{OO} \rightleftharpoons 2 \mathrm{OA}
$$

que l'on peut identifier à un équilibre chimique. La loi relative à cet équilibre, ou approximation quasi chimique [31, 32] donne la distribution relative à l'occupation des sites proches voisins :

$$
\frac{N_{\mathrm{AA}} N_{\mathrm{OO}}}{N_{\mathrm{OA}}^{2}}=\frac{1}{4} \exp \left(-\frac{\varepsilon}{k T}\right) \text {. }
$$

Une fois obtenue par la relation (4) la distribution relative des paires de sites, il est nécessaire de relier ces grandeurs aux grandeurs élémentaires $N_{\mathrm{A}}$ et $N_{\mathrm{O}}$. Soit un réseau de sites à deux dimensions où le nombre de proches voisins est $z$. Le nombre de demi-liaisons entre sites proches voisins qui aboutissent sur un site occupé est donc $z N_{\mathrm{A}}$. Sachant que sur une paire de sites AA, deux demi-liaisons aboutissent sur un site occupé $A$, et que sur une paire de site OA, seule une demiliaison aboutit sur un site occupé A, on obtient :

$$
2 N_{\mathrm{AA}}+N_{\mathrm{OA}}=z N_{\mathrm{A}}
$$

et une relation similaire pour les sites inoccupés :

$$
2 N_{\mathrm{OO}}+N_{\mathrm{OA}}=z N_{\mathrm{O}} \text {. }
$$

Soit $N$ le nombre total de sites disponibles sur la surface. $\theta_{\mathrm{A}}=N_{\mathrm{A}} / N$ est le recouvrement en sites occupés et $1-\theta_{\mathrm{A}}=N_{\mathrm{o}} / N$ est le recouvrement en sites inoccupés. Puisque $z$ est le nombre de liaisons aboutissant sur un site, le nombre total de paires de sites disponibles à la surface du réseau cristallin vaut alors $z N / 2$, soit $\theta_{\mathrm{AA}}=2 N_{\mathrm{AA}} / z N, \theta_{\mathrm{OO}}=2 N_{\mathrm{OO}} / z N$ et $\theta_{\mathrm{OA}}=$ $N_{\mathrm{OA}} / z N$. Les relations (4), (5) et (6) se réduisent alors à :

$$
\begin{gathered}
\frac{\theta_{\mathrm{AA}} \theta_{\mathrm{OO}}}{\theta_{\mathrm{OA}}^{2}}=\exp \left(-\frac{\varepsilon}{k T}\right) \\
\theta_{\mathrm{OO}}+\theta_{\mathrm{OA}}=\theta_{\mathrm{O}} \\
\theta_{\mathrm{AA}}+\theta_{\mathrm{OA}}=\theta_{\mathrm{A}} .
\end{gathered}
$$

La résolution des équations (7) à (9) permet d'obtenir le recouvrement $\theta_{\mathrm{AA}}$ en paires de sites occupés, soit :

$$
\theta_{\mathrm{AA}}=\theta_{\mathbf{A}}-\frac{2 \theta_{\mathbf{A}}\left(1-\theta_{\mathbf{A}}\right)}{1+\left\{1-4 \theta_{\mathbf{A}}\left(1-\theta_{\mathbf{A}}\right)[1-\exp (-\varepsilon / k T)]\right\}^{1 / 2}}
$$

Cette relation (10) qui relie le recouvrement en paires de sites AA au recouvrement en sites occupés A, à laquelle ont abouti pour la première fois Goymour et King [33], peut être aussi obtenue sous une forme identique en utilisant l'approximation de Bethe [34]. Enfin, le calcul exact à une dimension [35] conduit à un résultat identique.

Il est intéressant pour la discussion d'examiner maintenant ce que devient cette relation (10) dans trois cas particuliers importants, à savoir les cas de la limite haute température (et interactions nulles), des interactions attractives fortes $(\varepsilon / k T \rightarrow-\infty)$ et des interactions répulsives fortes $(\varepsilon / k T \rightarrow+\infty)$. La relation 
(10) se réduit alors respectivement à :

$$
\begin{array}{ll}
\varepsilon / k T \rightarrow 0 & \theta_{\mathrm{AA}}=\theta_{\mathrm{A}}^{2} \\
\varepsilon / k T \rightarrow-\infty & \theta_{\mathrm{AA}}=\theta_{\mathrm{A}}
\end{array}
$$

Les résultats concernant ces trois cas limites sont représentés sur la figure 9. Si l'on considère d'abord le cas des interactions répulsives fortes, on constate que lorsque l'énergie d'interaction $\varepsilon$ est grande devant l'énergie thermique $k T$, on observe bien lorsque le recouvrement augmente, le mécanisme séquentiel d'adsorption de l'hypothèse 1 de notre modèle ainsi qu'il est représenté sur la figure 7. Si maintenant, $k T$ devient grand devant l'énergie d'interaction, on obtient alors une répartition aléatoire en $\theta_{\mathbf{A}}^{2}$ des paires de sites occupés (Eq. (11)). Le terme :

$$
\delta=1-\exp (-\varepsilon / k T)
$$

de l'expression (10) qui, dans le cas des interactions répulsives varie de zéro (désordre total) à l'unité (ordre total) apparaît donc comme le paramètre d'ordre à courte distance [36] qui régit la répartition des sites. Pour des valeurs intermédiaires du paramètre d'ordre $\delta$, on trouve alors des répartitions intermédiaires de celles représentées par les courbes $b$ et $c$ de la figure 9 : pour les faibles valeurs de recou-

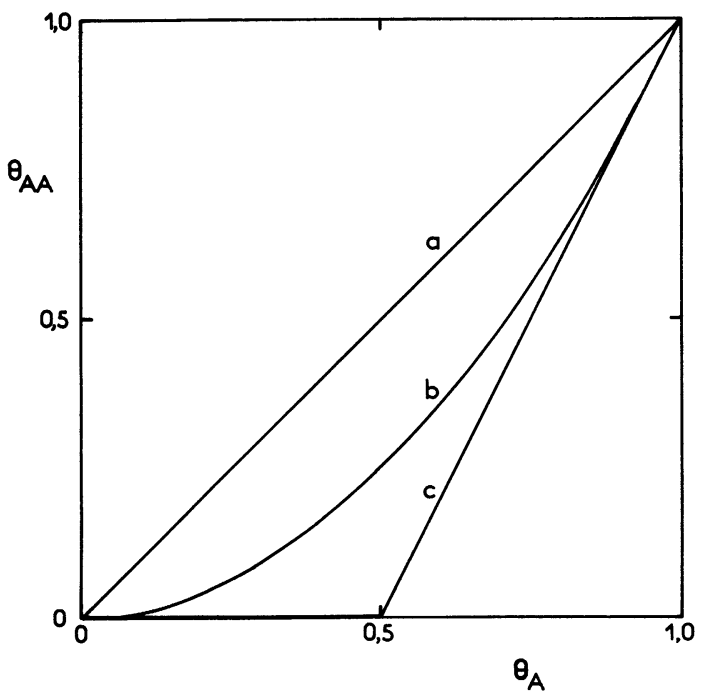

Fig. 9. - Evolution du recouvrement $\theta_{\mathrm{AA}}$ en paires de sites AA en fonction du recouvrement $\theta_{\mathrm{A}}$ en espèces $A$ dans le cas : a) des fortes interactions attractives $(\varepsilon / k T \rightarrow-\infty)$; b) des interactions nulles $(\varepsilon / k T \rightarrow 0)$; c) des fortes interactions répulsives $(\varepsilon / k T \rightarrow+\infty)$.

[Variation of surface coverage $\theta_{\mathrm{AA}}$ in sites AA, with surface coverage $\theta_{\mathrm{A}}$ in adspecies $\mathrm{A}:$ a) for large attractive interactions $(\varepsilon / k T \rightarrow-\infty) ;$ b) for no lateral interactions $(\varepsilon / k T \rightarrow 0)$; c) for large repulsive interactions $(\varepsilon / k T \rightarrow+\infty)$.] vrement, $\theta_{\mathrm{AA}}$ suit toujours une répartition en $\theta_{\mathrm{A}}^{2}$ :

$$
\theta_{\mathrm{AA}}=\theta_{\mathrm{A}}^{2} \exp (-\varepsilon / k T)
$$

tandis qu'aux forts recouvrements, on retrouve la répartition linéaire de l'équation (13). Pour un recouvrement unité $\left(\theta_{\mathrm{A}}=1\right)$, il y a alors égalité entre le recouvrement en sites occupés et le recouvrement en paires de sites occupés, soit $\theta_{\mathrm{A}}=\theta_{\mathrm{AA}}$. C'est la situation que l'on retrouve, cette fois quelle que soit la valeur du recouvrement $\theta_{\mathrm{A}}$, dans le cas des interactions attractives fortes (Eq. (12)). Ce résultat montre clairement l'établissement, dans ce cas, d'un ordre à grande distance conduisant à une séparation de phase : une première phase présentant un recouvrement unité telle que $\theta_{\mathrm{A}}=\theta_{\mathrm{AA}}=1$ et une seconde phase présentant un recouvrement nul tel que $\theta_{\mathrm{A}}=\theta_{\mathrm{AA}}=0$ (cf. Sect. 4). De même que pour les interactions répulsives, on retrouve, suivant la valeur de $\delta$, des répartitions intermédiaires de celles représentées par les courbes $a$ et $b$ de la figure 9 .

La conclusion principale qui ressort de cette discussion est qu'une condition nécessaire pour obtenir un recouvrement nul en paires de sites occupés est d'admettre des interactions répulsives fortes entre les espèces occupant deux sites proches voisins. Toutefois, ce recouvrement nul ne peut être atteint qu'au-dessous d'une valeur seuil du recouvrement en sites occupés (Eq. (14)). S'il est donc possible d'atteindre un recouvrement nul en paires de sites occupés, on a donc vérifié le bien-fondé, dans le cas de la gravure du silicium en plasma de gaz fluoré, d'une part de l'hypothèse 1 de notre modèle de gravure relative à l'adsorption séquentielle du fluor sur le silicium, d'autre part des hypothèses 3 et 4 qui définissent les conditions nécessaires à l'apparition de la gravure spontanée. De plus, les conclusions issues du développement analytique du modèle de phase adsorbée sont parfaitement corroborées par les résultats expérimentaux de la section 3, à savoir l'existence d'une anisotropie unité, liée à l'absence totale d'érosion latérale spontanée au-dessous d'une valeur seuil de recouvrement en fluor du silicium. Cette valeur seuil $\theta_{\mathrm{c}}$ au-dessous de laquelle on ne trouve plus deux groupements $\mathrm{SiF}_{2}$ proches voisins correspond en fait, pour le réseau carré du $\mathrm{Si}\langle 100\rangle$, à un recouvrement en fluor $\theta_{\mathrm{c}}=0,75$ (cf. Fig. 8).

Un autre point abordé dans la section 3 est la nécessité d'une diffusion en surface des espèces adsorbées, d'une part pour assurer le mécanisme séquentiel d'adsorption, hypothèse $1 \mathrm{du}$ modèle de gravure, d'autre part pour assurer l'équilibre indispensable de recouvrement entre les surfaces soumises au bombardement ionique et celles qui n'y sont pas soumises. Compte tenu de l'importance de la diffusion dans les mécanismes de gravure, il est utile, à partir du modèle analytique développé et discuté dans cette section, d'examiner les effets des interactions entre proches voisins sur la diffusion en surface. D'une manière 
générale, le coefficient de diffusion $D$ s'écrit :

$$
D=D_{0} \exp (-E / k T)
$$

où $D_{0}$ est la constante de diffusion et $E$ la hauteur de la barrière pour la diffusion ou énergie d'activation. L'influence des interactions répulsives ou attractives entre proches voisins sur l'énergie d'activation est schématisée sur la figure 10 . Soit $E_{0}$ l'énu.gie d'activation d'un adatome isolé $\left(\theta_{\mathrm{A}}=0\right)$. En présence d'un proche voisin, la hauteur de la barrière d'énergie est réduite dans le cas d'interactions répulsives et accrue dans le cas d'interactions attractives [37]. L'effet est supposé additif et donc proportionnel au nombre d'interactions avec les proches voisins.

Il en résulte donc que l'énergie d'activation ne peut prendre, pour un adatome donné, que certaines valeurs discrètes liées au nombre de sites proches voisins occupés. Dans le cas qui nous intéresse, celui des fortes interactions répulsives, il est possible d'analyser (Annexe I) l'évolution de l'énergie d'activation $E$ en fonction du taux de recouvrement $\theta_{\mathrm{A}}$. Cette évolution est représentée sur la figure 11 . On constate que, dans le cas d'un réseau carré, il existe quatre domaines de recouvrement, qui correspondent chacun à une valeur constante de l'énergie d'activation. Lorsque l'on passe d'un domaine au suivant, l'écart entre les énergies d'activation est $\varepsilon$.
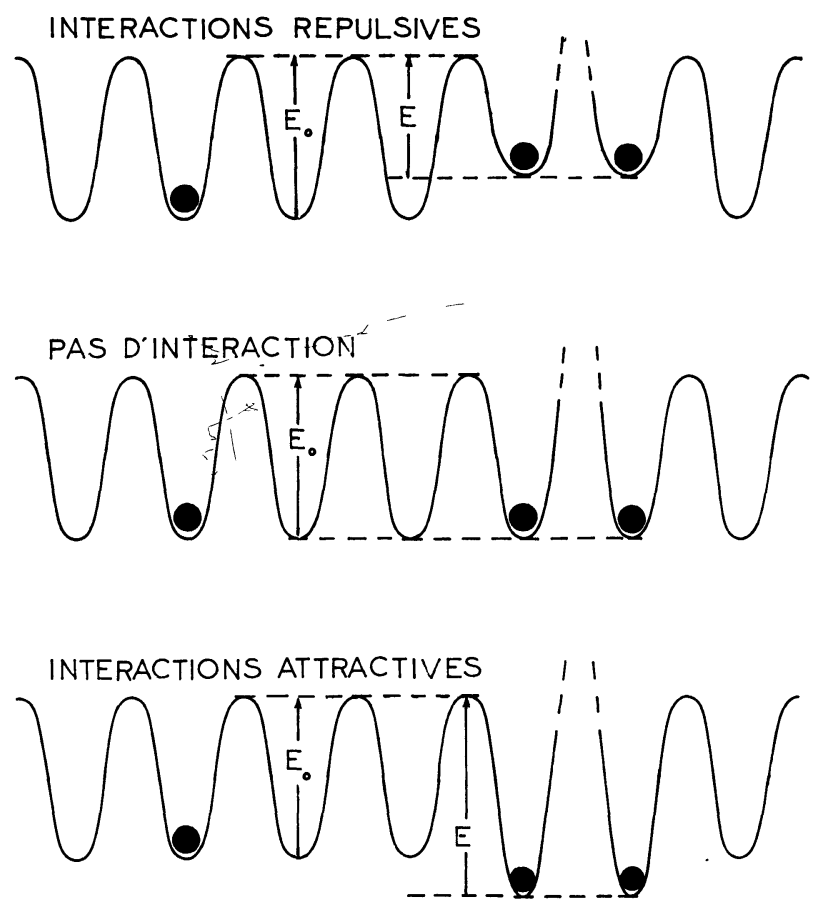

Fig. 10. - Influence de la nature des interactions entre proches voisins sur l'énergie d'activation $E$.

[Influence of lateral interactions between chemisorbed atoms in nearest neighbour positions on the activation energy barrier $E$.]
Le coefficient de diffusion $D$ présente donc une discontinuité pour les valeurs de recouvrement correspondant au passage d'un domaine à l'autre. Ainsi la diminution brutale de l'énergie d'activation au-dessus de $\theta_{\mathrm{A}}=0,5$ conduit à un accroissement important du coefficient de diffusion, lié à l'apparition de paires de sites proches voisins occupés.

Nous avons montré dans cette section que l'hypothèse d'interactions répulsives fortes entre groupes proches voisins adsorbés est cohérente avec le modèle de gravure spontané proposé dans la section précédente. Le rôle du bombardement ionique n'a pas jusqu'à présent été abordé. Nous nous proposons donc, dans la section 6, avant d'examiner les conséquences du modèle sur la gravure du silicium en plasma fluoré, de préciser les hypothèses concernant la gravure induite.

\section{Conséquences pour la gravure.}

L'étude analytique du modèle de phase adsorbée effectuée dans la section 5 a montré clairement, dans le cas d'interactions répulsives fortes entre proches voisins, l'établissement d'un ordre à courte distance qui permet de justifier les hypothèses de notre modèle, à savoir, mécanisme séquentiel d'adsorption sur la surface et absence de gravure spontanée au-dessous d'un certain seuil de recouvrement. Dans le cas de la gravure du silicium en plasma de gaz fluoré, il faut donc que le recouvrement en fluor soit supérieur à $\theta_{\mathrm{c}}=0,75$ pour trouver deux groupes $\mathrm{SiF}_{2}$ sur deux sites proches voisins. Dans ce cas, on observe bien la formation de $\mathrm{SiF}_{4}$ par la réaction (2), la seule possible du point de vue thermodynamique [38]. Le taux de réaction étant proportionnel au recouvrement en paires de sites

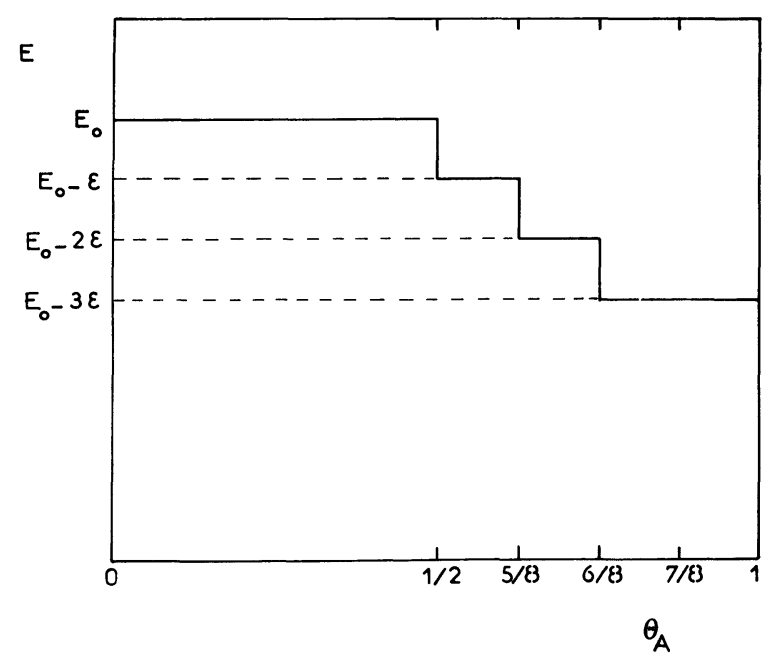

Fig. 11. - Evolution de l'énergie d'activation $E$ en fonction du taux de recouvrement de la surface pour un réseau carré (cas des interactions répulsives fortes).

[Evolution of the activation energy $E$ as a function of surface coverage $\theta_{\mathrm{A}}$ for a square lattice (large repulsive interactions).] 
proches voisins occupés, il présente, dans le cas des fortes interactions répulsives, une dépendance linéaire avec le taux de recouvrement en fluor pour $\theta>\theta_{\mathrm{c}}$. On a alors le comportement typique d'une réaction d'ordre 1. Si $\theta$ est le recouvrement effectif, le taux de réaction est porportionnel à $\theta-\theta_{c}$ (Eq. (13) et Fig. 8).

Dans le cas d'une gravure présentant une anisotropie unité, où la vitesse de gravure latérale spontanée est strictement nulle, le recouvrement en $\mathrm{SiF}_{2}$ est alors inférieur à 0,5 soit $\theta<0,75$. Seule subsiste la gravure induite par le bombardement ionique. $\mathrm{Si}$ l'on admet, toujours dans ce cas, que la formation du $\mathrm{SiF}_{4}$ observé ne peut résulter que de la réaction entre deux groupes $\mathrm{SiF}_{2}$ proches voisins, alors il devient évident que l'un des effets du bombardement ionique est de détruire l'ordre à courte distance instauré sur la surface par les interactions répulsives. Le transfert d'énergie par échange de quantité de mouvement opéré entre l'ion bombardant la surface et un groupe $\mathrm{SiF}_{x}$ permet à au moins un des atomes de fluor de franchir la barrière d'énergie pour la diffusion et de former une paire de groupes $\mathrm{SiF}_{2}$ proches voisins.

En réalité, à ce premier effet du bombardement ionique peuvent s'ajouter deux autres effets, à savoir le franchissement des deux barrières d'activation, de hauteur très disproportionnées que sont d'une part la chaleur de désorption $L_{0}$ des espèces $\mathrm{SiF}_{x}$ adsorbées, d'autre part l'énergie d'activation $R_{0}$ de la réaction (2) impliquant deux groupes $\mathrm{SiF}_{2}$ proches voisins (Fig. 12).

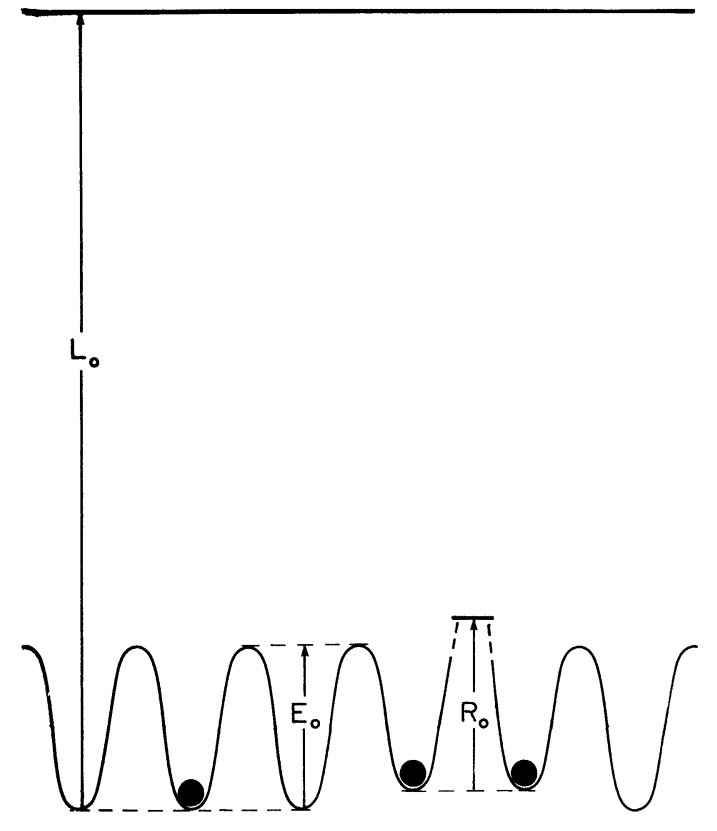

Fig. 12. - Représentation schématique de la chaleur de désorption $L_{0}$, de l'énergie d'activation $E_{0}$ à la diffusion et de la chaleur de réaction $R_{0}$ entre 2 groupes $\mathrm{SiF}_{2}$ proches voisins.

[Schematic representation of desorption heat $L_{0}$, activation energy barrier to surface migration $E_{0}$ and reaction heat $R_{0}$ for $\mathrm{SiF}_{2}$ radicals in nearest neighbour positions.]
Les trois effets décrits précédemment ont chacun leur domaine privilégié. La désorption directe des espèces $\mathrm{SiF}_{2}$ (ou plus généralement $\mathrm{SiF}_{x}$ ), qui ne conduit pas à un produit stable du point de vue thermodynamique et par conséquent la moins probable, implique à la fois une énergie suffisamment élevée des ions incidents et un appauvrissement de la surface en fluor, comme semblent le montrer les résultats expérimentaux de la section 3 . Les barrières d'activation liées d'une part à la diffusion, d'autre part à la réaction (2) soit $E$ et $R_{0}$, sont, elles, nettement moins élevées que la chaleur de désorption tout en étant du même ordre de grandeur entre elles puisque diffusion et réactions à la surface du silicium sont activées thermiquement à la température ambiante. Dans le cas de recouvrements en fluor proches de l'unité, seule l'activation de la réaction (2) est nécessaire à la gravure induite. Enfin, pour des recouvrements inférieurs au recouvrement critique $\theta<\theta_{\mathrm{c}}$, deux cas peuvent se présenter : si $E$ est supérieur à $R_{0}$, le franchissement de la barrière d'activation $E$ suffit, d'une part à accéder en situation de proche voisin, d'autre part à réagir; si $R_{0}$ est supérieur à $E$, le franchissement de la barrière d'activation $R_{0}$ est suffisant non seulement pour réagir, mais aussi pour accéder préalablement en position de proche voisin.

Quel que soit le mécanisme responsable de la gravure induite, il est logique d'admettre que le taux de collisions efficaces conduisant à un produit de réaction désorbé est proportionnel au produit du courant d'ions et de la densité de fluor adsorbé sur la surface. Si l'on suppose la désorption directe d'espèces $\mathrm{SiF}_{x}$ négligeable, on peut de plus admettre, en première approximation, des sections efficaces équivalentes pour l'activation de la cinétique de réaction et de la diffusion.

Maintenant précisées puis admises les hypothèses concernant la gravure spontanée et la gravure induite, il est possible d'établir un modèle de diffusion pour la gravure plasma, l'adsorption des réactants à partir de la phase gazeuse et la désorption des produits de réaction formés sur la surface étant contrôlée par la diffusion du fluor à la surface du silicium. En régime permanent, l'équation générale de la diffusion peut s'écrire :

$$
\sum_{i=1,2} \frac{\partial}{\partial x_{i}}\left(D \frac{\partial \sigma}{\partial x_{i}}\right)+v-\rho=0
$$

où $v$ est le flux de fluor adsorbé et $\rho$ le flux de fluor inclus dans les produits de réaction désorbés. $\sigma$ est la densité de sites d'adsorption occupés liée au recouvrement $\theta=\sigma / \sigma_{0}, \sigma_{0}$ étant la densité de sites d'adsorption pour le fluor.

Compte tenu des résultats de la section 5 , il faut distinguer deux cas : s'il n'existe aucune paire de sites occupés, il n'y a pas de gravure spontanée et seul le terme de gravure induite subsiste. S'il en existe, il y a gravure spontanée et les deux termes de gravures 
induite et spontanée coexistent. A ces deux cas, il est nécessaire d'associer des coefficients de diffusion distincts, soit respectivement $D^{-}$et $D^{+}$qui sont des constantes dans l'hypothèse des interactions fortes (cf. Sect. 5). L'équation (17) prend alors les formes suivantes :

$$
\begin{array}{ll}
\sigma>\sigma_{\mathrm{c}} & D^{+} \nabla^{2} \sigma+v=\eta j \sigma+\frac{\sigma-\sigma_{\mathrm{c}}}{\tau} \\
\sigma<\sigma_{\mathrm{c}} & D^{-} \nabla^{2} \sigma+v=\eta j \sigma
\end{array}
$$

où $\eta$, constante réactionnelle ionique, est un coefficient incluant la section efficace d'activation des espèces adsorbées et la stoechiométrie de composition des produits désorbés, $\tau$ le temps d'adsorption du fluor avant désorption spontanée sous forme de produit de réaction et où $\sigma_{\mathrm{c}}$ est relié au recouvrement critique en fluor $\theta_{\mathrm{c}}=\sigma_{\mathrm{c}} / \sigma_{0}$.

Examinons maintenant le cas d'un modèle de gravure réaliste comportant des zones soumises au bombardement ionique (gravure verticale $: j \neq 0$ ) et des zones qui n'y sont pas soumises (gravure latérale : $j=0$ ). Les équations de diffusion s'écrivent alors, dans le cas d'un modèle unidimensionnel (traits parallèles de longueur infinie) :

$$
\begin{aligned}
& \sigma>\sigma_{\mathrm{c}}\left\{\begin{array}{l}
D^{+} \frac{\mathrm{d}^{2} \sigma}{\mathrm{d} x^{2}}+v=\eta j \sigma+\frac{\sigma-\sigma_{\mathrm{c}}}{\tau} \\
D^{+} \frac{\mathrm{d}^{2} \sigma}{\mathrm{d} x^{2}}+v=\frac{\sigma-\sigma_{\mathrm{c}}}{\tau} \quad(j=0)
\end{array}\right. \\
& \sigma<\sigma_{\mathrm{c}}\left\{\begin{array}{l}
D^{-} \frac{\mathrm{d}^{2} \sigma}{\mathrm{d} x^{2}}+v=\eta j \sigma \\
D^{-} \frac{\mathrm{d}^{2} \sigma}{\mathrm{d} x^{2}}+v=0 \quad(j=0)
\end{array}\right.
\end{aligned}
$$

La continuité dans les deux couples d'équations s'obtient aisément en écrivant la continuité de la densité de sites d'adsorption occupés à la limite des zones bombardées et non bombardées. Essayons tout d'abord, sans résoudre le système dans le cas le plus général, d'exploiter ces équations dans quelques cas particuliers simples. Pour cela il est toutefois nécessaire d'établir auparavant l'expression du flux de fluor qui s'adsorbe à la surface du silicium.

Jusqu'à présent, et en particulier lors du développement analytique de l'adsorption (Sect. 5) nous avons supposé le recouvrement compris entre 0 et 1 . Dans cette hypothèse, notre modèle s'apparente alors à celui de Langmuir-Hinshelwood. Le flux de fluor adsorbé est alors donné par le modèle d'adsorption de Langmuir, soit :

$$
v=k p_{\mathrm{F}}(1-\theta)
$$

où $p_{\mathrm{F}}$ est la pression partielle de fluor atomique dans la phase vapeur (plasma de gaz fluoré) et $k$ le coefficient d'adsorption.
Considérons maintenant le cas d'une surface présentant un recouvrement homogène et soumise à un bombardement ionique uniforme. C'est le cas en particulier de la grille d'extraction du spectromètre de masse plongée dans le plasma (Sect. 3). Les équations qui régissent l'adsorption et la désorption sur la surface se réduisent alors à :

$$
\begin{array}{ll}
\sigma>\sigma_{\mathrm{c}} . & k p_{\mathrm{F}}(1-\theta)=\sigma_{0} \eta j \theta+\frac{\sigma_{0}\left(\theta-\theta_{\mathrm{c}}\right)}{\tau} \\
\sigma<\sigma_{\mathrm{c}} & k p_{\mathrm{F}}(1-\theta)=\sigma_{0} \eta j \theta .
\end{array}
$$

L'intensité du pic $\mathrm{SiF}_{3}^{+}$mesurée, correspondant à la désorption du produit de réaction $\mathrm{SiF}_{4}$, est l'image directe de la vitesse de gravure de la grille, et ce, quel que soit le régime, isotrope ou anisotrope. Afin de comparer ces résultats à ceux des profils de gravure obtenus dans les mêmes conditions sur des échantillons masqués, il est nécessaire de supposer que les coefficients de diffusion $D^{-}$et $D^{+}$sont suffisamment importants pour que les longueurs de diffusion soient grandes devant les épaisseurs à graver et que celles-ci sont faibles devant les dimensions des motifs. Dans ce cas, on peut alors considérer le recouvrement comme quasiment uniforme.

L'anisotropie, telle qu'elle est définie par la relation (1) s'écrit alors :

$$
A=1-\frac{\rho(j=0)}{\rho(j \neq 0)}
$$

soit, pour $\theta>\theta_{\mathbf{c}}$ :

$$
A=\frac{\eta j \theta}{\eta j \theta+\frac{\theta-\theta_{\mathrm{c}}}{\tau}}=\frac{1}{1+\frac{1}{\eta j \tau}\left(1-\frac{\theta_{\mathrm{c}}}{\theta}\right)} .
$$

On remarque alors que, à densité de courant constante, l'anisotropie décroît pour des valeurs croissantes de recouvrement. Lorsque la pression partielle de fluor atomique croit, le recouvrement $\theta$ tend alors rapidement vers la valeur unité et l'anisotropie vers une valeur constante.

Examinons maintenant comment évolue corrélativement la vitesse de gravure verticale en fonction de la pression au-delà du recouvrement critique. D'après l'équation (25), le flux de produits désorbés $\rho$, proportionnel à la vitesse de gravure est donné par :

$$
\rho=\frac{k p_{\mathrm{F}}\left(\eta j+1 / \tau-\theta_{\mathrm{c}} / \tau\right)}{k p_{\mathrm{F}} / \sigma_{0}+1 / \tau+\eta j} .
$$

Lorsque $p_{\mathrm{F}}$ augmente et tend vers l'infini, $\theta$ tend vers la monocouche et $\rho$ tend alors, en même temps que l'anisotropie, vers une valeur constante donnée par l'équation (29). Or, on constate sur la figure 5 que, la vitesse de gravure verticale, tout comme l'intensité du pic $\mathrm{SiF}_{3}^{+}$, continue de croître. En d'autres termes, il apparaît clairement que la cinétique d'adsorption choisie n'est pas conforme au modèle d'adsorption 
de Langmuir. Il faut donc rechercher un autre modèle, compatible du point de vue physique avec nos résultats expérimentaux et ceux issus de la littérature.

La mise en évidence, par de nombreux auteurs, de l'incorporation de fluor dans le silicium $[3,23,24]$ sous forme chimisorbée [25] montre qu'il est possible d'atteindre des recouvrements dépassant, et de beaucoup, la monocouche $[3,23,24]$. Un modèle d'adsorption qui permet de rendre compte d'un empilement similaire de couches chimisorbées est le modèle bien connu de B.E.T. (Brunauer-Emmett-Teller) qui conduit à l'isotherme du même nom (Annexe II).

Bien que ce point n'ait pas lieu d'être précisé dans les hypothèses de base du modèle de B.E.T., l'adsorption du fluor sur le silicium s'écarte du modèle classique en ce sens que, une fois la première couche remplie, l'adsorption ne se poursuit pas vers l'extérieur, mais vers l'intérieur, dans le volume du silicium par pénétration du fluor dans le réseau [39]. Autrement dit, les couches ne s'empilent pas les unes sur les autres, mais les unes sous les autres. Cette approche est en fait beaucoup plus réaliste que l'approche classique, car l'adsorption de la première couche ainsi que des suivantes fait appel au même type de liaison chimique. Dans le cas du silicium, l'adsorption du fluor dans les couches sous-jacentes s'effectue à travers des liaisons covalentes Si-F [25]. La phase adsorbée qui en résulte est parfaitement ordonnée, et seul un bombardement d'ions très énergétiques $(>400 \mathrm{eV})$ peut alors rendre la surface polycristalline [40].

Un point important est de savoir si les résultats de la section 5 , qui supposent un recouvrement inférieur à la monocouche, sont affectés par l'introduction du modèle d'adsorption de B.E.T. La réponse est négative pour peu que la chaleur d'adsorption pour les couches sous-jacentes soit inférieure de quelques $k T$ à la chaleur d'adsorption correspondant à la couche initiale. Dans ce cas, la formation de la première sous-couche ne s'effectue qu'une fois la couche initiale quasiment complète (Annexe II) et les conclusions de la section 5 restent alors valables. Par contre, les expressions des flux désorbés, induit et spontané, calculées pour $\theta<1$, ne peuvent être considérées, pour $\theta>1$, que comme une extrapolation.

De même que pour les modèles d'adsorption les plus étudiés, la cinétique d'adsorption relative au modèle de B.E.T. s'obtient aisément à partir des considérations de mécanique statistique utilisées pour l'établissement de l'isotherme [41]. Généralement, dans le modèle de B.E.T., on suppose qu'il est possible d'atteindre un nombre de couches adsorbées infini. Ceci ne semble pas être le cas du fluor avec le silicium $[23,24]$. Si l'on fait donc l'hypothèse que le nombre maximum de couches qu'il est possible d'adsorber est $s$, on obtient alors l'isotherme de B.E.T. à $s$ couches. Dans ce cas, la cinétique d'adsorption prend la forme simple (cf. Annexe II) :

$$
v=k p_{\mathrm{F}} \frac{s-\theta}{s} .
$$

Si $s=1$, on retrouve le cas de l'isotherme de Langmuir (Eq. (24)) et si $s$ tend vers l'infini, on obtient la cinétique du modèle de B.E.T.

Les équations (20) à (23) s'écrivent alors sous la forme définitive suivante :

$$
\begin{aligned}
& \sigma>\sigma_{\mathrm{c}}\left\{\begin{array}{l}
D^{+} \frac{\mathrm{d}^{2} \sigma}{\mathrm{d} x^{2}}+k p_{\mathrm{F}} \frac{s-\theta}{s}=\eta j \sigma+\frac{\sigma-\sigma_{\mathrm{c}}}{\tau} \\
D^{+} \frac{\mathrm{d}^{2} \sigma}{\mathrm{d} x^{2}}+k p_{\mathrm{F}} \frac{s-\theta}{s}=\frac{\sigma-\sigma_{\mathrm{c}}}{\tau}(j=0)
\end{array}\right. \\
& \sigma<\sigma_{\mathrm{c}}\left\{\begin{array}{l}
D^{-} \frac{\mathrm{d}^{2} \sigma}{\mathrm{d} x^{2}}+k p_{\mathrm{F}} \frac{s-\theta}{s}=\eta j \sigma \\
D^{-} \frac{\mathrm{d}^{2} \sigma}{\mathrm{d} x^{2}}+k p_{\mathrm{F}} \frac{s-\theta}{s}=0 \quad(j=0)
\end{array}\right.
\end{aligned}
$$

et les équations (25) et (26) deviennent :

$$
\begin{array}{ll}
\sigma>\sigma_{\mathrm{c}}, & k p_{\mathrm{F}} \frac{s-\theta}{s}=\sigma_{0} \eta j \theta+\frac{\sigma_{0}\left(\theta-\theta_{\mathrm{c}}\right)}{\tau} \\
\sigma<\sigma_{\mathrm{c}}, & k p_{\mathrm{F}} \frac{s-\theta}{s}=\sigma_{0} \eta j \theta
\end{array}
$$

où $\sigma_{0}$ est la densité de sites d'adsorption en fluor de la première couche.

L'expression de l'anisotropie reste inchangée, soit :

$$
\begin{array}{ll}
\sigma>\sigma_{\mathrm{c}}, & A=\frac{1}{1+\frac{1}{\eta j \tau}\left(1-\frac{\theta_{\mathrm{c}}}{\theta}\right)} \\
\sigma<\sigma_{\mathrm{c}}, & A=1 .
\end{array}
$$

Examinons, dans le cadre du modèle d'adsorption de B.E.T. à $s$ couches $(s \gg 1)$, les comportements de l'anisotropie et du flux de produits de réaction désorbés. Pour $\theta>\theta_{\mathfrak{c}}$, l'anisotropie décroît lorsque la pression de fluor atomique et donc le recouvrement $\theta$ augmentent. Dès que le recouvrement atteint quelques $\theta_{c}$, l'anisotropie décroît rapidement pour tendre vers une valeur limite donnée par la relation (28), soit :

$$
A=\frac{1}{1+\frac{1}{\eta j \tau}}
$$

C'est cette valeur de l'anisotropie qui est obtenue dans les plasmas de gravure classiques où la pression de travail dépasse généralement $5 \times 10^{-3}$ torr. La relation (38) montre que pour améliorer l'anisotropie dans ces plasmas " haute pression ", on dispose de trois possibilités, à savoir : i) augmenter $\tau$. Ceci peut être obtenu soit par un refroidissement efficace, soit par le choix d'un gaz dont la réactivité avec le silicium, comme c'est le cas du chlore, est inférieure à celle du fluor. C'est aussi le cas de la gravure du $\mathrm{SiO}_{2}$ par le fluor où $\tau$ est très grand et pour lequel seules des gravures anisotropes sont généralement obtenues; ii) 
augmenter $\eta$, c'est-à-dire la constante réactionnelle du bombardement ionique : on l'obtient généralement en augmentant l'énergie de bombardement vers le keV [7]; iii) augmenter la densité de courant $j$. Dans les décharges capacitives radiofréquence, un accroissement de la puissance injectée provoque l'augmentation simultanée de $j$ et $\eta[42,43]$.

L'évolution du flux $\rho$ des produits de réaction désorbés (pic $\mathrm{SiF}_{3}^{+}$et vitesse de gravure) en fonction du recouvrement est donnée directement par les équations (35) et (36) et tant que $\theta$ reste petit devant le nombre de couches maximum $s$, elles s'écrivent :

$$
\theta \ll s \begin{cases}\sigma>\sigma_{\mathrm{c}}, & \frac{k p_{\mathrm{F}}}{\sigma_{0}}=\eta j \theta+\frac{\theta-\theta_{\mathrm{c}}}{\tau} \\ \sigma<\sigma_{\mathrm{c}}, & \frac{k p_{\mathrm{F}}}{\sigma_{0}}=\eta j \theta\end{cases}
$$

et on constate alors d'une part que $\rho$ varie linéairement avec le recouvrement et d'autre part qu'il est proportionnel à la pression partielle de fluor atomique dans le plasma. Si de plus on suppose une variation linéaire, à puissance de décharge donnée, de la pression partielle de fluor atomique avec la pression totale du plasma, l'intensité du pic $\mathrm{SiF}_{3}^{+}$et la vitesse de gravure doivent présenter une variation linéaire avec la pression du plasma. En réalité, l'évolution de la pression de fluor atomique mesurée dans un plasma de gaz fluoré n'est pas tout à fait linéaire puisqu'elle varie moins vite que la pression totale du gaz introduit [17]. Cest bien ce que l'on observe, du moins dans le domaine de pression exploré, sur la figure 13 où les résultats de la figure 5 sont représentés sur une échelle log-log. Pour les pressions plus élevées, pour lesquelles le recouvrement $\theta$ n'est plus négligeable devant $s$, le flux de produit désorbé s'écrit, à partir de l'équation (35) :

$$
\rho=k p_{\mathrm{F}} \frac{\eta j+\frac{1}{\tau}-\frac{\theta_{\mathrm{c}}}{\tau s}}{\frac{k p_{\mathrm{F}}}{\sigma_{0} s}+\frac{1}{\tau}+\eta j} .
$$

Le flux de produits de réaction désorbés croît donc avec la pression pour tendre asymptotiquement vers la valeur finie :

$$
\rho=s \sigma_{0}\left[\eta j+\frac{1}{\tau}-\frac{\theta_{\mathrm{c}}}{\tau s}\right] .
$$

L'ensemble de ces résultats montre donc que, à densité de courant constante, l'accroissement de la vitesse de gravure avec la pression est suivie d'une saturation. Il faut toutefois noter que cette saturation s'obtient pour des valeurs de pression très supérieures à celles correspondant à la saturation de l'anisotropie. Dans ce cas, pour des valeurs $\tau$ et $\eta$ données, seule une augmentation de la densité de courant permet d'accroître encore la vitesse de gravure.

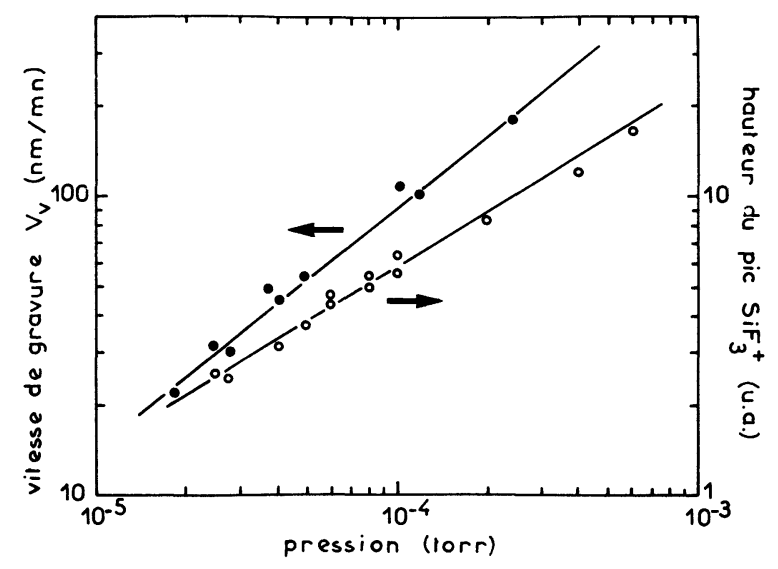

Fig. 13. - Evolution de la vitesse de gravure $V_{\mathrm{v}}$ et de la hauteur du pic $\mathrm{SiF}_{3}^{+}$en fonction de la pression de $\mathrm{SF}_{6}$.

[Etch rate and $\mathrm{SiF}_{3}^{+}$peak intensity as a function of $\mathrm{SF}_{6}$ pressure.]

Par contre, si une anisotropie unité est exigée, il est indispensable de travailler dans le domaine des pressions plus faibles où $\theta<\theta_{\mathrm{c}}$. La vitesse de gravure est alors considérablement réduite. Au recouvrement critique $\theta_{c}$, correspondent une pression partielle critique de fluor $p_{c}$ et une densité de courant d'ions critique $j_{\mathrm{c}}$ nécessaires à l'obtention de l'anisotropie unité. $\mathrm{La}$ relation qui relie ces grandeurs, issue de l'équation (39) est :

$$
j_{\mathrm{c}}=\frac{k p_{\mathrm{c}}}{\eta \sigma_{0} \theta_{\mathrm{c}}} .
$$

Elle montre une relation linéaire entre la pression partielle de fluor et la densité de courant critique $j_{\mathrm{c}}$. $\mathrm{Si}$ on admet, toujours en première approximation que dans le plasma, la pression partielle de fluor atomique est proportionnelle à la pression de travail du gaz fluoré, on en déduit donc, que pour se maintenir en régime de gravure anisotrope $\left(\theta<\theta_{c}\right)$ lorsque la pression de travail est accrue, il est nécessaire d'augmenter la densité de courant ionique, et par conséquent la densité de plasma, dans des proportions similaires. La vitesse de gravure anisotrope est accrue, elle aussi dans le même rapport (Eq. (40)). Par conséquent, en régime anisotrope $\left(\theta<\theta_{c}\right)$, une augmentation de la vitesse de gravure s'obtient par une augmentation de la pression de travail du plasma et une augmentation corrélative de sa densité.

Enfin, dernier résultat évident, mais qu'il est nécessaire de souligner, l'homogénéité de gravure ne peut être atteinte que dans des plasmas homogènes non seulement en densité mais aussi en espèces neutres réactives.

Dans cette section, nous avons montré comment évoluent les principaux paramètres de gravure en fonction des paramètres plasma, puis nous avons vérifié que les évolutions calculées ne sont pas en contradiction avec nos résultats expérimentaux. 
Cependant, afin de rendre plus concrets nos résultats, il apparaît nécessaire de les illustrer par quelques exemples numériques. Ceci fait l'objet de la dernière section.

\section{Résultats numériques et confirmations expérimen- tales.}

Dans cette dernière section, nous avons jugé intéressant de montrer comment, à partir de notre modèle analytique de gravure et des résultats expérimentaux, il était possible d'estimer les grandeurs principales qui régissent la gravure, à savoir le rendement réactionnel ionique $\eta$, le temps caractéristique de cinétique chimique $\tau$, le coefficient d'adsorption $k$, les coefficients de diffusion et les longueurs de diffusion correspondantes. Bien entendu, il n'est pas question de déterminer les valeurs précises de ces paramètres, mais plutôt leur ordre de grandeur.

Le rendement réactionnel ionique $\eta$, qui détermine le flux de silicium désorbé peut être facilement relié à la vitesse de gravure. Si $u$ est le nombre de couches de silicium érodées par seconde et $d$ l'épaisseur d'une couche, la vitesse de gravure s'écrit :

$$
V_{\mathrm{v}}=u d .
$$

Le flux d'atomes de silicium désorbés correspondants est égal au produit du nombre de couches désorbées par seconde par le nombre $M$ d'atomes de silicium dans la couche. Si les produits de réaction sont désorbés sous forme de $\mathrm{SiF}_{4}$, le flux de fluor correspondant vaut :

$$
\rho=4 u M=4 \frac{V_{\mathrm{v}} M}{d} .
$$

Pour la valeur du recouvrement critique $\theta_{\mathrm{c}}$ correspondant au passage au régime anisotrope, on peut écrire, compte tenu des équations (39) ou (40) :

$$
\eta=\frac{4 V_{\mathrm{c}} M}{d j_{\mathrm{c}} \theta_{\mathrm{c}} \sigma_{0}}
$$

où $V_{c}$ est la vitesse de gravure au recouvrement critique. Le rendement $Y$ en atomes de silicium désorbés par ion incident, grandeur couramment utilisée, s'écrit d'une manière générale :

$$
Y=\frac{e \rho}{4 j}=\frac{e V_{\mathrm{v}} M}{j d}
$$

où $e$ est la charge élémentaire.

Pour le recouvrement critique, $Y$ prend la valeur :

$$
Y=\frac{e \eta \theta_{\mathrm{c}} \sigma_{0}}{4}=\frac{e V_{\mathrm{c}} M}{j_{\mathrm{c}} d} .
$$

Application numérique :

Pour un cristal de silicium orienté $\langle 100\rangle, \sigma_{0} / 2=$ $M=6,8 \times 10^{18} \mathrm{~m}^{-2}$ et $d=1,36 \times 10^{-10} \mathrm{~m}$. Les échantillons sont de type $\mathrm{n}$ et de résistivité $2-3 \Omega . \mathrm{cm}$.
Pour le recouvrement critique $\theta_{c}=0,75$, la densité de courant est $j_{\mathrm{c}}=5 \mathrm{~A} \cdot \mathrm{m}^{-2}$ et la vitesse de gravure verticale mesurée est $V_{\mathrm{c}}=7,4 \times 10^{-10} \mathrm{~m} \mathrm{~s}^{-1}$ (Figs. 4 et 5).

On obtient alors : $\eta=2,9 \mathrm{~A}^{-1} \mathrm{~s}^{-1} \mathrm{~m}^{2}$ et $Y=1,2$.

Ce rendement est faible par rapport aux rendements rencontrés lors de la gravure assistée par faisceaux d'ions de forte énergie de l'ordre du keV [22]. Toutefois, dans le domaine des basses énergies, la constante réactionnelle ionique chute considérablement $[7,27]$, si bien que la valeur obtenue avec des ions de $70 \mathrm{eV}$ est tout à fait plausible.

Une fois $\eta$ calculé, l'ordre de grandeur du temps caractéristique d'adsorption $\tau$ peut être obtenu directement à partir de la valeur limite de l'anisotropie donnée par la relation (38), soit :

$$
\tau=\frac{A}{\eta j(1-A)} .
$$

Bien que l'évolution de l'anisotropie en fonction de la pression de $\mathrm{SF}_{6}$ n'ait pas été mesurée à courant constant, on peut supposer que, pour les pressions les plus élevées, la valeur limite de l'anisotropie est atteinte. L'application numérique est immédiate (avec $A=0,86$ et $j=1,6 \mathrm{~A} / \mathrm{m}^{2}$ ) et conduit à $\tau=1,3 \mathrm{~s}$.

La méthode d'évaluation du coefficient d'adsorption $k$ consiste, à partir d'une hypothèse, à vérifier si celle-ci est sensée ou absurde. Supposons donc pour le fluor atomique un coefficient de collage unité. A faible recouvrement $(\theta \ll s)$, le flux aléatoire de fluor atomique frappant le silicium est alors totalement adsorbé. Compte tenu de cette hypothèse, le flux de fluor désorbé sous forme de $\mathrm{SiF}_{4}$ est égal au flux de fluor atomique adsorbé, soit :

$$
\frac{p_{\mathrm{F}}}{\left(2 \pi m_{\mathrm{F}} k T\right)^{1 / 2}}=n_{\mathrm{F}}\left(\frac{k T}{2 \pi m_{\mathrm{F}}}\right)^{1 / 2}=\eta j \theta \sigma_{0}
$$

où $m_{\mathrm{F}}$ représente la masse d'un atome de fluor. L'application numérique de l'équation (50) effectuée pour $\theta=\theta_{c}$ conduit à un flux de fluor adsorbé de $1,5 \times$ $10^{20} \mathrm{~m}^{-2} \mathrm{~s}^{-1}$. Ce flux correspond à une densité de fluor atomique dans le plasma $n_{\mathrm{F}}=10^{18} \mathrm{~m}^{-3}$. En fait, les valeurs ainsi calculées sont des valeurs par excès, car à $\theta=\theta_{\mathrm{c}}, \mathrm{SiF}_{4}$ n'est plus le seul produit désorbé (cf. Fig. 6). Cependant, ce résultat n'est pas en contradiction avec la valeur obtenue par titration en spectrométrie de masse [17] dans un plasma de $\mathrm{SF}_{6}$ excité par RCE à une pression légèrement plus élevée, mais à puissance de décharge plus faible. Dans notre dispositif expérimental, le taux important de fluor atomique est corroboré par l'observation, lors de l'excitation du plasma, d'une augmentation non négligeable de la pression totale due à la dissociation de $\mathrm{SF}_{6}$. On peut donc conclure qu'un coefficient de collage unité est parfaitement plausible dans le cas de l'adsorption du fluor atomique par le silicium. Un tel résultat s'explique parfaitement par la valeur élevée de la chaleur d'adsorption du fluor sur le silicium. Le coeffi- 
cient d'adsorption s'écrit alors :

$$
k=\left(2 \pi m_{\mathrm{F}} k T\right)^{-1 / 2} .
$$

Si l'on admet ce résultat, la pression partielle de fluor atomique dans le plasma peut donc se déduire, dans le domaine des faibles recouvrements $(\theta \ll s)$ et donc des faibles pressions, directement de la vitesse de gravure observée. La comparaison avec le taux de fluor mesuré par actinométrie devrait permettre, soit d'infirmer, soit de confirmer notre hypothèse et/ou la méthode d'actinométrie employée.

Les dernières grandeurs qui restent à calculer sont les coefficients et les longueurs de diffusion du fluor à la surface du silicium. L'équation de diffusion (31) peut se mettre sous la forme :

$$
D \frac{\mathrm{d}^{2} \sigma}{\mathrm{d} x^{2}}-\frac{\sigma}{t}=-f
$$

en posant :

$$
\begin{aligned}
& \frac{1}{t}=\eta j+\frac{1}{\tau}+\frac{v_{0}}{\sigma_{0} s} \\
& f=v_{0}+\frac{\sigma_{\mathrm{c}}}{\tau}
\end{aligned}
$$

et

$$
v_{0}=k p_{\mathbf{F}} \text {. }
$$

La solution générale de cette équation s'écrit :

$$
\sigma=A \exp \left(\frac{x}{\Lambda}\right)+B \exp \left(-\frac{x}{\Lambda}\right)+f t
$$

où

$$
\Lambda=(D t)^{1 / 2}
$$

représente la longueur de diffusion. $A$ et $B$ sont des constantes qui dépendent des conditions aux limites.

Le premier résultat important qui en résulte est que, à chaque coefficient de diffusion $D^{-}$et $D^{+}$, intervenant dans les équations (31) à (34), il correspond deux longueurs de diffusion distinctes, à savoir :

$$
\begin{aligned}
\sigma<\sigma_{\mathrm{c}} & {\left[\begin{array}{l}
\Lambda^{-}=\left(\frac{D^{-}}{\eta j+\frac{v_{0}}{\sigma_{0} s}}\right)^{1 / 2} \\
\Lambda_{0}^{-}=\left(\frac{D^{-} \sigma_{0} s}{v_{0}}\right)^{1 / 2}
\end{array}\right.} \\
\sigma>\sigma_{\mathrm{c}} & {\left[\begin{array}{l}
\Lambda^{+}=\left(\frac{D^{+}}{\eta j+\frac{1}{\tau}+\frac{v_{0}}{\sigma_{0} s}}\right)^{1 / 2} \\
\Lambda_{0}^{+}=\left(\frac{D^{+}}{\frac{1}{\tau}+\frac{v_{0}}{\sigma_{0} s}}\right)^{1 / 2}
\end{array}\right.}
\end{aligned}
$$

Dans ces équations, les indices + et - sont liés aux indices correspondants des coefficients de diffusion, tandis que l'indice 0 indique un courant de bombardement ionique nul (zones non bombardées). Comme on peut le constater, les longueurs de diffusion dépendent de très nombreux paramètres et par conséquent le seul commentaire immédiat que l'on peut apporter, concerne leur valeur relative. $\mathrm{Si}$, dans un régime de gravure donné, isotrope ou anisotrope, on compare les longueurs de diffusion dans les zones soumises au bombardement ionique et celles qui n'y sont pas soumises, on voit que l'effet du courant est de provoquer une réduction de la longueur de diffusion. De plus, si l'on suppose que $D^{+} \gg D^{-}$(Sect. 5), on vérifie que les longueurs de diffusion (58) à (61) sont classées par valeurs croissantes, soit :

$$
\Lambda^{-}<\Lambda_{0}^{-}<\Lambda^{+}<\Lambda_{0}^{+} .
$$

Il est toutefois possible de pousser plus loin l'analyse en utilisant certaines particularités des équations générales de la diffusion dans le domaine des faibles recouvrements $(\theta \ll s)$ et plus particulièrement dans le domaine du régime de gravure anisotrope $\left(\theta<\theta_{\mathrm{c}}\right)$. Dans les zones non soumises au bombardement ionique, l'équation de diffusion (34) prend alors la forme simple :

$$
D^{-} \frac{\mathrm{d}^{2} \sigma}{\mathrm{d} x^{2}}+v_{0}=0 .
$$

La solution générale s'écrit dans ce cas particulier :

$$
\sigma=-\frac{v_{0}}{2 D^{-}} x^{2}+C x+E .
$$

Dans les zones soumises au bombardement ionique, l'équation (33) prend la forme simplifiée :

$$
D^{-} \frac{\mathrm{d}^{2} \sigma}{\mathrm{d} x^{2}}-\eta j \sigma=-v_{0}
$$

dont la solution générale est :

$$
\begin{aligned}
\sigma=A \exp \left[\frac{x}{\left(D^{-} / \eta j\right)^{1 / 2}}\right]+ \\
\quad+B \exp \left[-\frac{x}{\left(D^{-} / \eta j\right)^{1 / 2}}\right]+\frac{v_{0}}{\eta j} .
\end{aligned}
$$

Considérons maintenant le cas de deux zones semiinfinies soumises au bombardement ionique et séparées par une bande de largeur $2 a$ qui n'y est pas soumise (Fig. 14). Ce cas peut être assimilé en fait à un cas de gravure classique : un fond de gravure soumis au bombardement ionique, une paroi latérale non soumise au bombardement ionique, puis la surface de l'échantillon protégée par un masque. La comparaison suppose que le masque protecteur possède, pour la diffusion du fluor en surface, les mêmes propriétés que le 

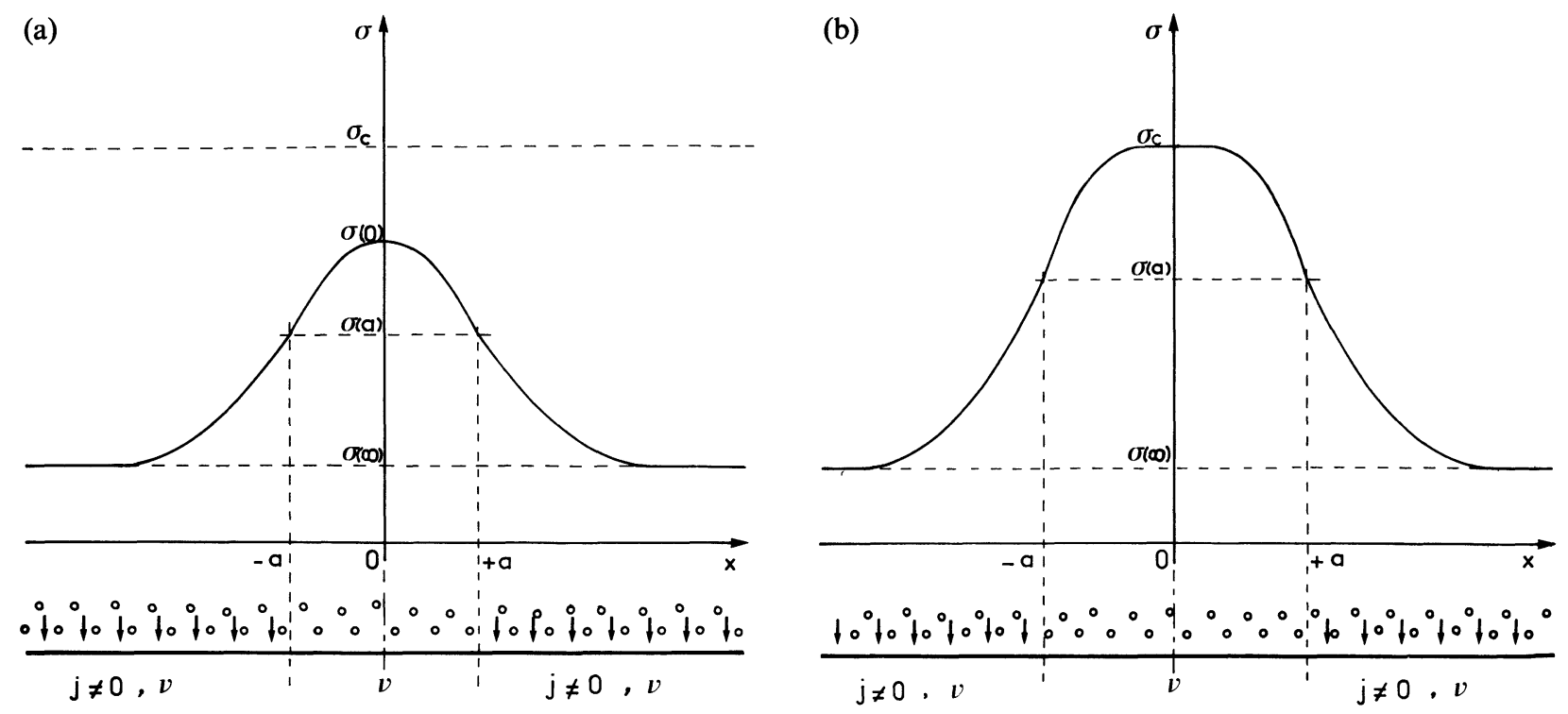

Fig. 14. - Evolution spatiale de la densité de sites occupés sur deux surfaces semi-infinies soumises au bombardement ionique et séparées par une bande de largeur $2 a$ pour des densités en fluor $\sigma(0)$ au centre de cette bande : a) inférieures à $\left.\sigma_{\mathrm{c}} ; \mathrm{b}\right)$ supérieures à $\sigma_{\mathrm{c}}$.

[Spatial evolution of fluorine density $\sigma$ on two semi-infinite planes subjected to ion bombardment and separated by a $2 a$ wide strip for two values of the fluorine density in the middle of the strip : a) $\sigma(0)<\sigma_{c}$; b) $\sigma(0)>\sigma_{\mathrm{c}}$ ]

silicium. On suppose en outre que les caractéristiques plasma d'une part restent inchangées tout au long de la gravure, d'autre part sont telles que le recouvrement, homogène sur toute la surface à l'instant initial, est inférieur au recouvrement critique. Pendant toute la durée de la gravure, la vitesse de gravure verticale prise à l'infini étant supposée constante, la hauteur $2 a$ de la paroi latérale croît linéairement avec le temps. Tant que le recouvrement au milieu de la paroi reste inférieur au recouvrement critique, la gravure spontanée ne s'amorce pas et la gravure reste anisotrope. Au-delà du recouvrement critique, la gravure spontanée s'amorce. Si on poursuit le processus, très rapidement la gravure spontanée a lieu sur une hauteur importante de la paroi latérale (Fig. 14b). De plus, au-delà de $\theta_{\mathrm{c}}$, on change de régime de diffusion. Les longueurs de diffusion augmentent alors considérablement (Inégalité (62)) si bien que le recouvrement peut être considéré comme constant (Fig. 14b). Dans ce qui suit, on se propose de développer analytiquement ce cas de gravure et de montrer comment, à partir des caractéristiques de la gravure observées lors de l'apparition de la gravure latérale spontanée, il est possible d'estimer le coefficient de diffusion $D^{-}$.

La fonction $\sigma(x)$ étant paire, nous nous intéresserons seulement aux abscisses positives (Fig. 14). A partir des équations (64) et (66), les conditions aux limites permettent de calculer $\sigma(x)$ :

$$
\begin{array}{ll}
0 \leqq x \leqq a & \sigma=-\frac{v_{0}}{2 D^{-}}\left(x^{2}-a^{2}\right)+\frac{v_{0}}{\eta j}+\frac{v_{0} a}{\left(\eta j D^{-}\right)^{1 / 2}} \\
a \leqq x & \sigma=\frac{v_{0} a}{\left(\eta j D^{-}\right)^{1 / 2}} \exp \left[-\frac{x-a}{\left(D^{-} / \eta j\right)^{1 / 2}}\right]+\frac{v_{0}}{\eta j} .
\end{array}
$$

On en déduit aisément :

$$
\sigma(0)-\sigma(\infty)=\frac{v_{0} a^{2}}{2 D^{-}}+\frac{v_{0} a}{\left(\eta j D^{-}\right)^{1 / 2}} .
$$

L'équation (69) montre, comme on pouvait s'y attendre, que plus le recouvrement de départ $\sigma(\infty)$ est faible, plus la hauteur de la paroi latérale que l'on peut atteindre sans apparition de la gravure spontanée est grande. Si $a_{\mathrm{c}}$ est la demi-hauteur de paroi latérale pour laquelle l'apparition de la gravure spontanée est constatée, on a alors, compte tenu de l'équation (69) :

$$
\frac{\Delta \sigma}{a_{\mathrm{c}} v_{0}} D^{-}-\frac{a_{\mathrm{c}}}{2}=\left(\frac{D^{-}}{\eta j}\right)^{1 / 2}=\Lambda^{-}
$$


en posant :

$$
\Delta \sigma=\sigma_{\mathrm{c}}-\sigma(\infty)
$$

$\mathrm{Si}$ on ne retient que la solution physique :

$D^{-}=\frac{1}{\eta j}\left(\frac{v_{0} a_{\mathrm{c}}}{2 \Delta \sigma}\right)^{2}\left[1+\left(1+\frac{2 \eta j \Delta \sigma}{v_{0}}\right)^{1 / 2}\right]^{2}$.

Afin d'obtenir la plus grande valeur possible de $a_{\mathrm{c}}$, nous nous sommes placés dans des conditions expérimentales extrêmes, à savoir à la pression de travail la plus basse possible, soit $2,75 \times 10^{-5}$ torr de $\mathrm{SF}_{6}$. La vitesse de gravure est alors très lente et la durée de l'expérience élevée. Les résultats sont présentés sur la figure 15. Outre un pied de gravure important dû probablement aux effets de paroi sur les ions, on constate que la gravure reste anisotrope jusqu'à $2 a_{\mathrm{c}}=15 \mu \mathrm{m}$ environ.

\section{$20 \mu \mathrm{m}$}

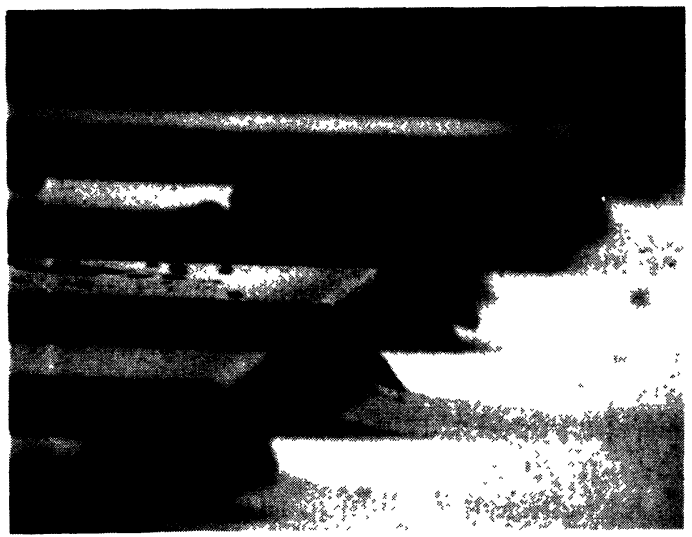

\section{$40 \mu \mathrm{m}$}

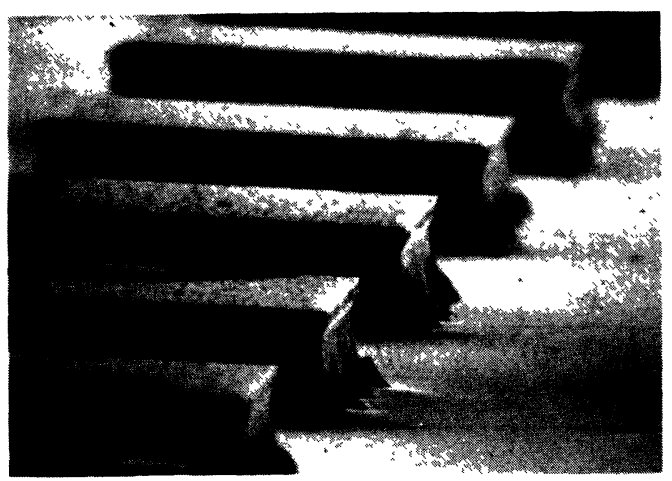

Fig. 15. - Photographies réalisées au MEB d'échantillons de silicium masqués aluminium et gravés en plasma de $\mathrm{SF}_{6}$ à $2,75 \times 10^{-5}$ torr, $600 \mathrm{~W}$. Profondeur gravée : a) $11,5 \mu \mathrm{m}$; b) $18,6 \mu \mathrm{m}$.

[SEM views of silicon wafers masked with aluminium and etched in an $\mathrm{SF}_{6}$ plasma $\left(p=2.75 \times 10^{-5}\right.$ torr, $P_{\mathrm{UHF}}=$ $600 \mathrm{~W}$ ). The etch depth is : a) $11.5 \mu$, b) $18.6 \mu \mathrm{m}$.]
Application numérique :

La valeur du flux de fluor adsorbé et celle du recouvrement $\theta(\infty)$ peuvent être calculées à partir de la vitesse de gravure verticale mesurée et des équations (40) et (45), soit :

$$
v_{0}=\frac{4 V_{\mathrm{v}} M}{d}=\eta j \sigma_{0} \theta(\infty)
$$

Pour $p_{\mathrm{SF}_{6}}=2,75 \times 10^{-5}$ torr, $V_{\mathrm{v}}=3,8 \times 10^{-10} \mathrm{~m} \cdot \mathrm{s}^{-1}$ et $j=5 \mathrm{~A} \cdot \mathrm{m}^{-2}$, on obtient :

$$
v_{0}=7,6 \times 10^{19} \mathrm{~m}^{-2} \cdot \mathrm{s}^{-1} \text { et } \theta(\infty)=0,4 .
$$

La valeur du coefficient de diffusion $D^{-}$calculée à partir de l'équation (72) vaut :

$$
D^{-}=1,8 \times 10^{-9} \mathrm{~m}^{2} \cdot \mathrm{s}^{-1}
$$

et la longueur de diffusion calculée à partir de l'équation (70) vaut alors :

$$
\Lambda^{-}=11 \mu \mathrm{m} \text {. }
$$

Cette application numérique, bien que délicate, a le mérite de dégager l'ordre de grandeur de deux paramètres fondamentaux intervenant dans la gravure. En particulier, on voit que la plus courte des longueurs de diffusion définies précédemment est de l'ordre de la dizaine de microns dans nos conditions expérimentales. Cette longueur de diffusion est généralement bien plus grande que toutes les épaisseurs à graver, ce qui justifie l'hypothèse sur les longueurs de diffusion de la section 6 .

Nous avons calculé les ordres de grandeur des paramètres qui régissent les phénomènes de gravure dans le cas du silicium en plasma fluoré $\mathrm{SF}_{6}$. Nous avons vérifié que le modèle proposé était cohérent non seulement sur le plan phénoménologique, mais aussi sur un plan plus quantitatif. Nous en avons tiré des conclusions générales directement utilisables en gravure et ce, quel que soit le type de réacteur utilisé. Il reste cependant à confronter ce modèle à d'autres résultats issus de la littérature et à estimer la généralité du modèle.

En premier lieu, nous avons choisi de confronter notre modèle au cas de la gravure réactive assistée par faisceaux d'ions. L'exemple de la gravure du silicium par $\mathrm{XeF}_{2}$ assistée par des ions argon d'énergie $1 \mathrm{keV}$ $[8,22]$ est celui qui se compare le mieux à la gravure du silicium en plasma $\mathrm{SF}_{6}$ que nous avons étudiée : d'une part la chimisorption dissociative de $\mathrm{XeF}_{2}$ sur le silicium ne nécessite pas la dissociation préalable de la molécule, d'autre part, les ions qui induisent la gravure ne sont pas réactifs, tout comme dans un plasma de $\mathrm{SF}_{6}$ où les ions $\mathrm{SF}_{x}^{+}$sont majoritaires. Seule l'énergie est fondamentalement différente (1 keV d'un côté, $70 \mathrm{eV}$ de l'autre).

L'évolution du rendement de gravure $Y$, rapport du nombre d'atomes de silicium désorbés par ion incident 
est illustrée sur la figure 3 de la référence [22]. A densité de courant constante, pour les faibles flux de gaz incident, la variation de $Y$ est linéaire. Pour les flux élevés, on observe la saturation du rendement $Y$. Cest bien le comportement attendu, compte tenu des résultats de la section 6. D'après notre modèle, à partir des équations (42) et (47), le rendement à saturation s'écrit :

$$
Y=\frac{e \rho}{4 j} \# \frac{e \sigma_{0} s\left(\eta j+1 / \tau-\theta_{c} / \tau s\right)}{4 j} .
$$

On constate donc que le rendement $Y$ maximum obtenu à la saturation dépend fortement de la valeur de $s$. Dans le plasma de $\mathrm{SF}_{6}$ étudié, le domaine de pression exploré n'a pas permis d'observer cette saturation et donc d'estimer la valeur de $s$.

Une autre confirmation de notre modèle est suggérée par l'étude des premières étapes de la fluoruration du silicium par photoémission synchrotron [44]. En particulier, les auteurs ont pu mesurer l'abondance relative des espèces fluorées $\mathrm{SiF}, \mathrm{SiF}_{2}$ et $\mathrm{SiF}_{3}$ présentes à la surface du silicium après chimisorption dissociative de $\mathrm{XeF}_{2}$. Ils constatent alors que $\mathrm{SiF}$ et non pas $\mathrm{SiF}_{2}$ est l'espèce prédominante à la surface du silicium. Ils en déduisent que les modèles de gravure existants sont pour le moins incomplets puisqu'ils font généralement intervenir, pour la plupart, des réactions de surface entre groupes $\mathrm{SiF}_{2}$. "Ironiquement, disent-ils, $\mathrm{SiF}_{2}$ n'est pas l'espèce majoritaire sur les surfaces étudiées ". Or, dans le cas de notre modèle, il est clair qu'il ne peut subsister sur la surface deux groupes $\mathrm{SiF}_{2}$ proches voisins sans qu'ils réagissent pour former $\mathrm{SiF}_{4}$. Notre modèle implique donc que le taux de $\mathrm{SiF}_{2}$ résiduel ne puisse excéder le taux de $\mathrm{SiF}$ présent sur la surface du silicium.

Tout au long de ce travail, nous avons choisi d'étudier l'exemple de la gravure du silicium en plasma de $\mathrm{SF}_{6}$. Un point important est de savoir si le modèle proposé peut s'étendre à d'autres systèmes chimiques comportant soit d'autres matériaux à graver que le silicium soit d'autres gaz réactifs que l'hexafluorure de soufre. S'il en est ainsi, il est alors possible de trouver pour ces systèmes des conditions de gravure permettant d'annuler la vitesse de gravure latérale $\left(V_{1}=0 / A=1\right)$ tout en conservant une vitesse de gravure verticale non négligeable. Un examen non exhaustif de la littérature montre que nombreux sont les exemples de systèmes présentant un tel comportement (cf. Tableau I). Ils concernent non seulement la gravure du silicium, mais aussi des métaux, et ce, aussi bien avec des gaz réactifs dilués qu'avec des mélanges plus complexes. On constate donc que le modèle mis au point dans le cas simple de la gravure du silicium en plasma $\mathrm{SF}_{6}$ semble pouvoir être généralisé à de très nombreux systèmes chimiques.

\section{Conclusion.}

L'objet de cette étude a consisté à proposer, à partir des résultats expérimentaux de la gravure du silicium en
Tableau I. - Exemples de systèmes chimiques mentionnés dans la littérature conduisant dans certaines conditions de gravure à l'obtention de profils anisotropes.

[Examples of chemical systems exhibiting a behaviour similar to the $\mathrm{Si} / \mathrm{F}$ system.]

\begin{tabular}{|l|c|c|}
\hline \multicolumn{1}{|c|}{ Mélange de gaz } & Substrat & Réf. \\
\hline \multirow{2}{*}{$\mathrm{SF}_{6} / \mathrm{Ar}$} & $\mathrm{Si}$ & {$[18]$} \\
\cline { 2 - 3 } & $\mathrm{W}$ & {$[40]$} \\
\hline $\mathrm{Cl}_{2} / \mathrm{Ar}$ & $\mathrm{Si} \mathrm{poly}$ & {$[41]$} \\
\hline \multirow{2}{*}{$\mathrm{SF}_{6} / \mathrm{O}_{2}$} & $\mathrm{Si} \mathrm{poly}$ & {$[42]$} \\
\hline & $\mathrm{MoSi}_{2}$ & {$[42]$} \\
\hline $\mathrm{SF}_{6} / \mathrm{Cl}_{2}$ & $\mathrm{Si} \mathrm{poly}$ & {$[41]$} \\
\hline $\mathrm{BCl}_{3} / \mathrm{Cl}_{2}$ & $\mathrm{Al}$ & {$[41]$} \\
\hline
\end{tabular}

plasma de $\mathrm{SF}_{6}$, un modèle cohérent de gravure par plasma. Les gravures ont été effectuées dans un Plasma Multipolaire Microonde excité à la Résonance Cyclotronique Electronique. L'évolution des paramètres de gravure, anisotropie et vitesse, et des produits de réaction analysés par spectrométrie de masse en transmission, en fonction de la pression de $\mathrm{SF}_{6}$, a permis de mettre en évidence le passage à un régime de gravure anisotrope en dessous d'une pression de $\mathrm{SF}_{6}$ critique. Ce passage s'accompagne de l'apparition de produits de réaction non saturés autres que $\mathrm{SiF}_{4}$, signe d'une diminution du recouvrement en fluor du silicium. La déduction essentielle de ces résultats expérimentaux est que la gravure spontanée, responsable du défaut d'anisotropie, apparaît seulement au-dessus d'un recouvrement critique du silicium par le fluor.

Afin de rendre compte complètement de ces résultats expérimentaux, nous avons donc été amenés à proposer nos propres hypothèses sur les mécanismes réactionnels mis en jeu dans la gravure plasma. Ainsi est-il nécessaire, pour donner une cohérence aux modèles existants, de leur adjoindre deux hypothèses supplémentaires fondamentales qui sont les suivantes :

1) existence de fortes interactions répulsives entre atomes de fluor adsorbés proches voisins;

2) diffusion en surface des espèces absorbées.

Une fois admises ces deux hypothèses, on obtient un modèle de gravure que l'on peut schématiser comme suit :

a) l'adsorption du fluor s'effectue par un mécanisme séquentiel; 
b) $\mathrm{SiF}_{4}$ est le seul produit de réaction de la gravure spontanée;

c) à la surface du silicium, le mécanisme réactionnel de gravure spontanée est :

$$
\mathrm{SiF}_{2} \text { (ads) }+\mathrm{SiF}_{2} \text { (ads) } \rightarrow \mathrm{Si}+\mathrm{SiF}_{4} ;
$$

d) la réaction n'est possible qu'entre deux groupes $\mathrm{SiF}_{2}$ plus proches voisins;

e) le bombardement ionique induit sur la surface du silicium la formation $\mathrm{de} \mathrm{SiF}_{4}$ par destruction de l'ordre à courte distance, et/ou par activation de la cinétique de réaction;

f) le bombardement ionique peut induire la désorption d'espèces non saturées $\operatorname{SiF}_{x}(x<4)$.

Enfin, pour être tout à fait complet, la cohérence de notre modèle de gravure avec les résultats expérimentaux impose une hypothèse supplémentaire, à savoir celle d'un modèle d'adsorption multicouches du type B.E.T. On vérifie alors que, dans le domaine de pression exploré, le flux aléatoire de fluor atomique présent dans le plasma frappant la surface de silicium est adsorbé dans sa quasi-totalité, indiquant par là même que le processus limitant de la cinétique de gravure est le taux de fluor dans le plasma.

Toutefois, tout au long de notre étude, on a supposé la constante réactionnelle ionique $\eta$ et le temps caractéristique de cinétique chimique $\tau$ constants. Si ces hypothèses sont plausibles dans un domaine de recouvrement peu étendu autour du recouvrement critique, il n'est pas assuré que leur extension aux forts recouvrements soit réaliste. Dans ce cas, bien que la cinétique de gravure ne devrait pas s'en trouver affectée, il peut en être tout autrement pour l'anisotropie et donc pour les profils de gravure observés. L'étude de l'évolution de l'anisotropie dans le domaine des fortes pressions, non accessibles dans notre dispositif expérimental, devrait alors permettre d'aider à combler cette lacune. Enfin, dans notre étude, l'énergie $W_{+}$des ions a été fixée à $70 \mathrm{eV}$. La connaissance de l'évolution de $\eta$ en fonction de l'énergie $W_{+}$doit permettre, elle aussi, d'inclure ce paramètre dans notre modèle. D'une manière générale, la connaissance détaillée de $\eta\left(\theta, W_{+}\right)$et $\tau(\theta)$ est un préalable à l'élaboration d'un modèle exhaustif de gravure par plasma.

\section{Remerciements.}

Les auteurs tiennent tout particulièrement à remercier M. Pichot et L. Vallier pour l'intérêt qu'ils ont manifesté à ce travail et leurs critiques constructives ainsi que A. Durandet pour son aide expérimentale lors de la gravure des tranchées dans le silicium et la patience qu'il a manifestée à cette occasion !

\section{Annexe I.}

EVOLUTION DU COEFFICIENT DE DIFFUSION EN FONCTION DU TAUX DE RECOUVREMENT DANS LE CAS D'INTERACTIONS RÉPULSIVES FORTES. - S'il existe des interactions entre espèces adsorbées sur un réseau, le calcul exact du coefficient de diffusion n'est généralement pas possible. Dans le cas des interactions répulsives fortes $(\varepsilon / k T \rightarrow \infty)$, il est toutefois possible d'introduire des conditions et des hypothèses supplémentaires, à savoir :

1) Le nombre d'interactions est minimum à taux de recouvrement donné;

2) la diffusion s'effectue à nombre d'interactions constant;

3) la diffusion est régie par les adatomes présentant le plus grand nombre d'interactions (ayant le plus grand nombre de proches voisins);

4) un adatome ne peut diffuser que vers un site inoccupé proche voisin.

$\mathrm{Si}$ les deux premiers points n'appellent pas de commentaires, il n'en est pas de même du point 3 : rappelons que le coefficient de diffusion $D$ s'écrit :

$$
D=D_{0} \exp (-E / k T)
$$

où $E$ est la barrière d'énergie pour la diffusion. Si $E_{0}$ est l'énergie d'activation d'un adatome isolé $\left(\theta_{\mathrm{A}}=0\right)$, celle-ci, en présence d'un proche voisin, est réduite de la valeur de l'énergie d'interaction $\varepsilon$ (Fig. 10). Si un second site proche voisin est occupé, elle est réduite de la valeur $2 \varepsilon$. L'énergie d'activation prend donc des valeurs discrètes liées au nombre $r$ de sites proches voisins occupés. Le rapport des coefficients de diffusion correspondant à deux valeurs successives $r+1$ et $r$ de l'énergie d'activation s'écrit :

$$
\frac{D_{\mathrm{r}+1}}{D_{\mathrm{r}}}=\exp \left(\frac{\varepsilon}{k T}\right)
$$

Dans le cas d'interactions fortes $(\varepsilon / k T \rightarrow \infty)$, on constate donc que la contribution à la diffusion des adatomes présentant un nombre d'interactions moindre est négligeable.

Enfin, si l'on suppose qu'un adatome ne peut diffuser que vers un site inoccupé, la valeur minimale que peut prendre l'énergie d'activation d'un adatome entouré de $z$ sites proches voisins est :

$$
E=E_{0}-(z-1) \varepsilon .
$$

Une fois admises les conditions et hypothèses 1 à 4 , il est possible de déterminer l'évolution des énergies d'activation et des coefficients de diffusion dans les cas d'un réseau unidimensionnel et celui du réseau carré.

Réseau unidimensionnel $(z=2)$. - Le cas du réseau unidimensionnel est particulièrement simple. Si $\theta_{\mathrm{A}}<0,5$, il n'y a pas d'interactions entre proches voisins et alors $E=E_{0}$. Si $\theta_{\mathrm{A}}>0,5$, la diffusion n'est possible que pour les adatomes ayant un site voisin inoccupé et donc $E=E_{0}-\varepsilon$.

Réseau carré $(z=4)$. - Le cas de ce réseau bidimensionnel est plus complexe car, d'une part les confi- 
gurations possibles dans une maille élémentaire en fonction du recouvrement sont nombreuses, d'autre part le processus de diffusion nécessite parfois, pour respecter les conditions et hypothèses 1 à 4 , le déplacement conjugué de deux adatomes. On suppose que ceci n'est possible que si l'énergie d'activation liée au deuxième adatome est inférieure à celle du premier, de telle sorte que la diffusion du second atome ne soit pas le processus limitant.

Comme dans le cas unidimensionnel, tant que $\theta_{\mathrm{A}}<0,5$, la diffusion se fait à nombre d'interactions nul et par conséquent $E=E_{0}$. Pour $\theta_{\mathrm{A}}>0,5$, les différentes configurations possibles sont représentées sur la figure 16 pour une maille élémentaire du réseau et pour des recouvrements croissants. Pour un taux de recouvrement moyen $1 / 2<\theta_{\mathrm{A}}<5 / 8$, la diffusion est assurée par des adatomes n'ayant qu'un site voisin occupé. L'énergie d'activation correspondante est $E=E_{0}-\varepsilon$. De même, on constate que pour $5 / 8<$ $\theta_{\mathrm{A}}<6 / 8$ et $6 / 8<\theta_{\mathrm{A}}<1$, l'énergie d'activation dans chacun de ces cas vaut respectivement $E=E_{0}-2 \varepsilon$ et $E=E_{0}-3 \varepsilon$.

\section{Annexe II.}

AdSORPTION MULTICOUCHES : MODÈLE DE BRUNAUEREmmet-Teller (B.E.T.). - Dans ce modèle [48], on considère un réseau bidimensionnel comportant $N_{0}$ sites discernables mais indépendants, sur lesquels viennent s'adsorber un nombre indéfini d'espèces, toutes de même nature, arrangées sur un nombre infini de couches. Ainsi, sur les $N_{0}$ sites de la surface sont adsorbés $N_{1}$ atomes qui constituent un nouvel ensemble de sites, sur lesquels viennent s'adsorber $\mathrm{N}_{2}$ atomes... De même les $N_{k}$ adatomes de la couche $k$ représentent $N_{k}$ sites d'adsorption potentiels pour les $N_{k+1}$ atomes qui forment la $(k+1)$-ième couche. Soit $q_{k}$ la fonction de partition d'un adatome de la couche $k$. Si les fonctions de partition des adatomes sur des couches d'ordre supérieur ou égal à 2 sont toutes égales à $q_{2}$, la fonction de partition grand canonique $\phi$ de l'ensemble constitué s'écrit [48] :

$$
\begin{aligned}
\phi=\left[1+q_{1} \lambda \sum_{k=0}^{\infty}\left(q_{2} \lambda\right)^{k}\right]^{N_{0}}= \\
=\left[\frac{1-q_{2} \lambda+q_{1} \lambda}{1-q_{2} \lambda}\right]^{N_{0}}
\end{aligned}
$$

où $\lambda$ représente l'activité absolue des adatomes.

Le taux de recouvrement, qui s'en déduit simplement, prend la forme :

$\theta=\frac{\lambda}{N_{0}}\left(\frac{\partial \log \phi}{\partial \lambda}\right)_{N_{0}, T}=\frac{c x}{(1-x+c x)(1-x)}$

avec $c=q_{1} / q_{2}$ et $x=q_{2} \lambda$ En écrivant l'égalité des activités dans la phase adsorbée et la phase gazeuse, l'équation (77) exprime aussi l'isotherme d'adsorption selon le modèle de B.E.T.

Considérons maintenant le cas particulier du sys- (a)

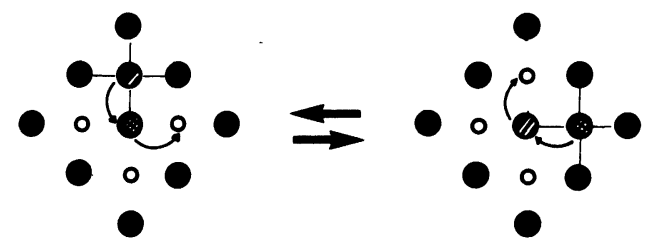

(b)

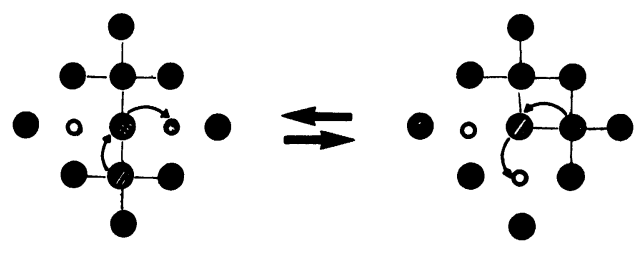

(c)
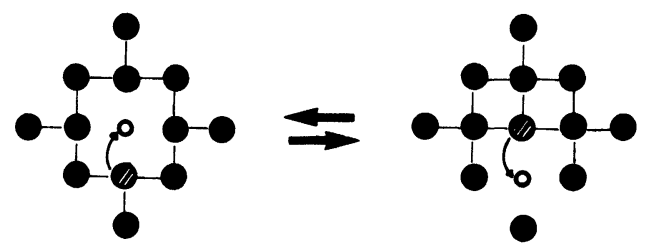

- - adatomes

- sites d'adsorption

Fig. 16. - Les différents cas (de figure) possibles pour la diffusion dans la maille élémentaire. Evolution en fonction du taux de recouvrement : a) $1 / 2<\theta_{\mathrm{A}}<5 / 8, E=E_{0}-\varepsilon$; b) $5 / 8<\theta_{\mathrm{A}}<6 / 8, E=E_{0}-2 \varepsilon$; c) $6 / 8<\theta_{\mathrm{A}}<1, E=$ $E_{0}-3 \varepsilon$.

[Possibilities of diffusion patterns in the elementary lattice mesh. Evolution as a function of surface coverage : a) $1 / 2<\theta_{\mathrm{A}}<5 / 8, E=E_{0}-\varepsilon ;$ b) $5 / 8<\theta_{\mathrm{A}}<6 / 8, E=$ $E_{0}-2 \varepsilon$; c) $6 / 8<\theta_{\mathrm{A}}<1, E=E_{0}-3 \varepsilon$.]

tème fluor/silicium pour lequel l'énergie d'adsorption du fluor atomique peut être estimée à environ $6 \mathrm{eV}$. Si l'on suppose que l'énergie d'adsorption qui correspond au remplissage de la seconde couche est quelque peu inférieure à l'énergie d'adsorption sur la couche superficielle, alors le coefficient $c$, rapport des fonctions de partition $q_{1}$ et $q_{2}$, devient très rapidement grand devant 1. Ainsi, à température ambiante, une différence d'énergie d'adsorption de l'ordre de $0,3 \mathrm{eV}$ conduit à $c=10^{5}$. L'isotherme correspondante est tracée sur la figure 17. On constate que, avec l'hypothèse faite, l'adsorption sur la seconde couche et les suivantes ne peut advenir qu'une fois la première couche remplie dans sa quasitotalité. Jusqu'à une valeur de $\theta$ voisine de l'unité, on suit donc l'isotherme de Langmuir (cf. Fig. 17).

Nous avons supposé jusqu'à présent que l'adsorption pouvait s'effectuer sur un nombre illimité de couches. Dans le cas où ce nombre est fini et égal à $s$, la fonction de partition $\phi$ est quelque peu modifiée :

$$
\begin{gathered}
\phi=\left[1+q_{1} \lambda \sum_{k=0}^{s-1}\left(q_{2} \lambda\right)^{k}\right]^{N_{0}}= \\
=\left[\frac{1-q_{2} \lambda+q_{1} \lambda-q_{1} q_{2}^{s} \lambda^{s+1}}{1-q_{2} \lambda}\right]^{N_{0}}
\end{gathered}
$$




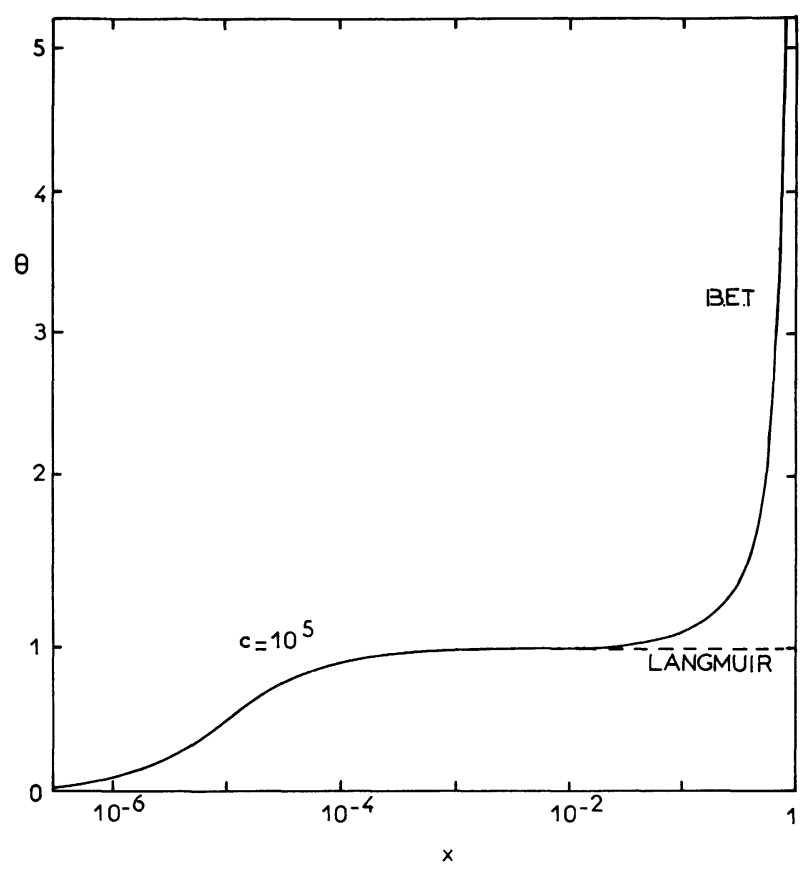

Figure 17. - Comparaison des isothermes d'adsorption de Langmuir et de B.E.T.

[Comparison of B.E.T. and Langmuir adsorption isotherms.]

de telle sorte que l'isotherme de B.E.T. à $s$ couches est décrite par :

$$
\theta=\frac{c x\left(1-(s+1) x^{s}+s x^{s+1}\right)}{\left(1-x+c x-c x^{s+1}\right)(1-x)} .
$$

On retrouve l'isotherme de B.E.T., lorsque $s$ tend vers l'infini et l'isotherme de Langmuir si $s=1$.

D’une manière générale, il est possible, à partir des considérations statistiques permettant l'établissement des isothermes, de déterminer les cinétiques d'adsorption et de désorption correspondantes [41]. Ces cinétiques reposent sur l'équilibre existant entre phase gazeuse et phase adsorbée. Il est décrit par :

$$
\frac{\mathrm{d} \theta}{\mathrm{d} t}=r_{\mathrm{a}}-r_{\mathrm{d}}
$$

où $r_{\mathrm{a}}$ et $r_{\mathrm{d}}$ désignent respectivement les taux d'adsorption et de désorption.

Pendant un laps de temps $\mathrm{d} t$, le nombre de molécules qui sont adsorbées est :

$$
r_{\mathrm{a}} \mathrm{d} t=k_{\mathrm{a}} p S \mathrm{~d} t
$$

où $k_{\mathrm{a}}$ représente le coefficient d'adsorption, $p$ la pression du composé dans la phase gazeuse considérée comme idéale et $S$ la fraction de la surface encore inoccupée à l'instant $t$. Pendant la même période $\mathrm{d} t$, le nombre d'adatomes qui désorbent de cette même surface est donné par :

$$
r_{\mathrm{d}} \mathrm{d} t=k_{\mathrm{d}} \lambda S \mathrm{~d} t
$$

$\lambda$ étant l'activité des adatomes dans la phase adsorbée et $k_{\mathrm{d}}$ le coefficient de désorption. En tenant compte des équations (81) et (82), on peut écrire (80) sous la forme :

$$
\frac{\mathrm{d} \theta}{\mathrm{d} t}=k_{\mathrm{a}} p S-k_{\mathrm{d}} \lambda S .
$$

Dans le cas d'un modèle d'adsorption à $s$ couches, la fraction $S$ de la surface inoccupée est $(s-\theta) / s$, l'activité absolue $\lambda$ pouvant se calculer à partir de l'isotherme d'adsorption. Si $s$ tend vers l'infini, on retrouve (Eq. (81)) la cinétique d'adsorption de la loi de Henry. Si $s=1$ on aboutit à la cinétique décrivant le modèle d'adsorption de Langmuir.

\section{Bibliographie}

[1] Chabert, P. et Pelletier, J., Secondes Journées d'Etude sur la Gravure Sèche en Microélectronique. Grenoble, 22-24 Nov. 1983, Le Vide les Couches Minces Suppl. 218 (1983) 25.

[2] Coburn, J. W., Plasma Chem. Plasma Proc. 2 (1982) 1.

[3] Winters, H. F., Coburn, J. W. and Chuang, T. J., J. Vac. Sci. Technol. B 1 (1983) 469.

[4] KIng, D. A. and Wells, M. G., Proc. R. Soc. Lond. A 339 (1974) 245.

[5] Flamm, D. L. and Donnelly, V. M., Plasma Chem. Plasma Process 1 (1981) 317.

[6] Coburn, J. W. and Winters, H. F., J. Appl. Phys. 50 (1979) 3189.

[7] Tu, Y. Y., Chuang, T. J. and Winters, H. F., Phys. Rev. B 23 (1981) 823.

[8] Gerlach-Meyer, U., Surf. Sci. 103 (1981) 524.

[9] Okano, H. and Horinke, Y., Jpn. J. Appl. Phys. 20 (1981) 2429.
[10] Mauer, J. L., Logan, J. S., Zielinski, L. B. and SchwartZ, G. C., J. Vac. Sci. Technol. 15 (1978) 1734.

[11] Arnal, Y., Pelletier, J., Рomot, C., Petit, B. et Durandet, A., Appl. Phys. Lett. 45 (1984) 132.

[12] Pelletier, J., Arnal, Y., Petit, B. and Pomot, C., Extended Abstract, Electrochem. Soc. 84-2 (1984) 544.

[13] Pomathiod, L., Debrie, R., Arnal, Y. and PelleTIER, J., Phys. Lett. 106A (1984) 301.

[14] Petit, B., Pelletier, J. and Molins, R., J. Electrochem. Soc. 132 (1985) 982.

[15] Eisele, K. M., J. Electrochem. Soc. 128 (1981) 123.

[16] Hirobe, K. and Azuma, H., J. Electrochem. Soc. 132 (1985) 938.

[17] Suzuki, K., Nishimatsu, S., Ninomiya, K. and OkudaIRA, S., Proc. Int. Ion Engineering Congress. ISIAT'83 and IPAT'83, Kyoto (1983) p. 1645. 
[18] Mahi, B., Arnal, Y., Pelletier, J. and Pomot, C., Proc. 5th International Colloquium on Plasmas and Sputtering, Antibes, June 10-14 (1985) p. 251.

[19] Foner, S. N. and Hudson, R. L., J. Chem. Phys. 25 (1956) 602.

[20] Chuang, T. J., J. Appl. Phys. 51 (1980) 2614.

[21] Morar, J. F., McFeely, F. R., ShinN, N. D., LandGREN, G. and Himpsel, F. J., Appl. Phys. Lett. 45 (1984) 174.

[22] Gerlach-Meyer, U., Coburn, J. W. and Kay, E., Surf. Sci. 103 (1981) 177.

[23] Brandt, W. W., Wagner, J. J. and Honda, T., J. Appl. Phys. 56 (1984) 1195.

[24] Frieser, R. G., Montillo, F. J., Zingerman, N. B., CHU, W. K. and MADER, S. R., J. Electrochem. Soc. 130 (1983) 2237.

[25] Roop, B., Joyce, S., Schultz, J. C., Shinn, N. D. and Steinfeld, J. I., Appl. Phys. Lett. 46 (1985) 1187.

[26] MCFeEly, F. R., Bull. APS 30 no 3 (1985) 461.

[27] MAYer, T. M., Barker, R. A. and WhitMan, L. J., J. Vac. Sci. Technol. 18 (1981) 349.

[28] Lagally, M. G., Lu, T. M. and Welkie, D. G., J. Vac. Sci. Technol. 17 (1980) 223.

[29] Gronwald, K. D. and Henzler, M., Surf. Sci. 117 (1982) 180.

[30] WAng, G. C. and Lu, T. M., Phys. Rev. Lett. 50 (1983) 2014.

[31] Guggenheim, E. A., Proc. R. Soc. A 148 (1935) 304.

[32] Hill, T. L., An Introduction to Statistical Thermodynamics (Addison-Wesley Publish Comp. Inc., London) Chapitre 14, 1960, p. 238.
[33] Goymour, C. G. and King, D. A., J. Chem. Soc. Faraday Trans. I 69 (1973) 749.

[34] Idem référence [32], Chapitre 14, p. 252.

[35] Pimbley, J. M. and Lu, T. M., J. Appl. Phys. 57 (1985) 1121.

[36] KIng, D. A., CRC Crit. Rev. Solid State Mater. Sci. 7 (1978) 167.

[37] Bowker, M. and KING, D. A., Surface Sci. 71 (1978) 583.

[38] Thermodata, Thermodynamical Data Bank, B.P. 66, 38402 St Martin d'Hères Cedex, France.

[39] Seel, M. and Bagus, P. S., Phys. Rev. B 28 (1983) 2023.

[40] Thomas, J. H., McGinn, J. T. and Hammer, L. H., Appl. Phys. Lett. 47 (1985) 746.

[41] Jaroniec, M. and Garbacz, J. K., Thin Solid Films 62 (1979) 237.

[42] Taillet, J., J. Physique Lett. 40 (1979) L-223.

[43] Pelletier, J., Arnal, Y., Petit, B., Рomot, C. and Pichot, M., J. Phys. D. 19 (1986) 795.

[44] McFeely, F. R., Morar, J. F., ShinN, N. D., LandGREN, G. and Himpsel, F., Phys. Rev. B 30 (1984) 764.

[45] Arnal, Y., Pelletier, J., Petit, B. and Рomot, C. (not published).

[46] Light, R. W. and Bell, H. B., Proc. of the 5th Symposium on Plasma Processing, Electrochem. Soc. 85-1 (1984) 430.

[47] Zhang, M., LI, J. Z., Adesida, I. and Wolf, E. D., J. Vac. Sci. Technol. B 1 (1983) 1037.

[48] Idem référence [32], Chapitre 7, p. 134. 